\title{
DYSON FERRARI-SPOHN DIFFUSIONS AND ORDERED WALKS UNDER AREA TILTS
}

\author{
DMITRY IOFFE, YVAN VELENIK, AND VITALI WACHTEL
}

\begin{abstract}
We consider families of non-colliding random walks above a hard wall, which are subject to a self-potential of tilted area type. We view such ensembles as effective models for the level lines of a class of $2+1$-dimensional discrete-height random surfaces in statistical mechanics. We prove that, under rather general assumptions on the step distribution and on the self-potential, such walks converge, under appropriate rescaling, to non-intersecting Ferrari-Spohn diffusions associated with limiting Sturm-Liouville operators. In particular, the limiting invariant measures are given by the squares of the corresponding Slater determinants.
\end{abstract}

\section{INTRODUCTION}

Random walks under area tilts mimic phase separation lines in certain lowtemperature two-dimensional lattice models of statistical mechanics, particularly in the regime of pre-wetting. A prototypical example is the two-dimensional Ising model in a large box with negative boundary conditions and a small positive magnetic field $h$. In such circumstances, the \pm -interface is pushed towards the boundary of the box and its fluctuations above flat segments of the boundary are expected to be of order $h^{-1 / 3}$. Rigorous justification of the latter claim is still an open problem (but see [21] for partial results in this direction). Instead, the papers [12, 15] are devoted to a refined analysis of effective random walk models of such interfaces. In particular, the full scaling limits were identified in [15], for a large class of effective random walks, as Ferrari-Spohn diffusions [10].

In this paper, we consider ensembles of $n$ non-colliding random walks which are subject to generalized area tilts. Precise definition are given in Section 3. These ensembles are intended to model non-intersecting level lines for certain lowtemperature $2+1$-dimensional interfaces (which themselves are intended to model two-dimensional random surfaces of lattice statistical mechanics). A prototypical example is the SOS model, see [3, 4] and references therein, or even more so its version with bulk Bernoulli fields which was introduced in [13]. In either case, lowtemperature level lines have the structure of Ising polymers whose effective random

Date: July 16, 2018.

DI was supported by the Israeli Science Foundation grant 1723/14.

YV was partially supported by the Swiss National Science Foundation. 
walk representation is discussed in [14] and is based on the general fluctuation theory of ballistic walks with self-interactions as developed in [16].

In Section 2, we introduce and briefly discuss the class of limiting objects, which we call Dyson Ferrari-Spohn diffusions. The latter can be alternatively described as Ferrari-Spohn diffusions conditioned to remain ordered, or as ergodic $n$-dimensional diffusions driven by the log-derivative of the Slater determinants of the corresponding Sturm-Liouville operators. The construction is well understood: We refer to [19, Section 2] for extensive details on determinantal random point fields in general and Fermi gas in particular, and to [7, Section 3] where such diffusions are discussed for specific kernels in the context of random matrix theory.

Properties of Dyson Ferrari-Spohn diffusions, in the form we need them, are formulated in Theorem 2.1. To keep our exposition self-contained and to stress the role played by the Karlin-McGregor formula, we sketch the proof.

Our effective model of ordered walks under area tilts is introduced in Section 3, while our main result, Theorem A, is formulated in Subsection 3.4. In Subsection 3.6, we introduce the rescaling notation which is employed in all the subsequent arguments. The step-by-step structure of our arguments is explained in Subsection 3.7. The details of the proofs are given in Sections 4-7 and in the Appendix. The organization of these sections is described in Subsection 3.8. Many of our technical estimates rely on strong approximation techniques and on a refinement of recent results on random walks in Weyl chambers and on cones [6, 8].

\section{Sturm-Liouville operators and Dyson Ferrari-Spohn Diffusions}

2.1. One particle. Consider the Sturm-Liouville operator

$$
\mathrm{L}=\frac{1}{2} \frac{\mathrm{d}^{2}}{\mathrm{~d} r^{2}}-q(r)
$$

where $q$ is a non-negative symmetric $C^{2}$-potential satisfying

$$
\lim _{|r| \rightarrow \infty} q(r)=\infty
$$

We can either think of $L$ as being defined on $\mathbb{L}_{2}\left(\mathbb{R}_{+}\right)$with zero boundary conditions at zero, or as being defined on $\mathbb{L}_{2}(\mathbb{R})$. It is classical fact [5] that $L$ has a complete orthonormal family of eigenfunctions

$$
\mathrm{L} \varphi_{i}=-\mathrm{e}_{i} \varphi_{i} \quad 0<\mathrm{e}_{1}<\mathrm{e}_{2}<\cdots \nearrow \infty .
$$

The Krein-Rutman eigenfunction $\varphi_{1}$ is positive on $(0, \infty)$, respectively on $\mathbb{R}$.

In the case of the half-line, $L$ has a closed self-adjoint extension from $C_{0}(0, \infty)$, whereas in the case of $\mathbb{R}$ it has a closed self-adjoint extension from $C_{0}(\mathbb{R})$. In both cases, the domain of the closure is given by

$$
\mathcal{D}(\mathrm{L})=\left\{f=\sum_{k} f_{k} \varphi_{k}: \sum_{k} \mathrm{e}_{k} f_{k}^{2}<\infty\right\} .
$$


We proceed to discuss the half-line case only; the full-line case would be a literal repetition.

$\mathrm{L}$ is a generator of a contraction semigroup $\mathrm{T}_{t}$ on $\mathbb{L}_{2}\left(\mathbb{R}_{+}\right)$: For $f=\sum f_{k} \varphi_{k}$,

$$
\mathrm{T}_{t} f(r)=\sum_{k} f_{k} \mathrm{e}^{-\mathrm{e}_{k} t} \varphi_{k}
$$

This semigroup has the following probabilistic representation: For $r>0$, let $\hat{\mathbf{P}}^{r}$ be the (sub-probability) path measure of the Brownian motion started at $r$ and killed upon hitting the origin. Then, for any $f \in \mathcal{D}(\mathrm{L})$ and any $t \geq 0$,

$$
\mathrm{T}_{t} f(r)=\hat{\mathbf{E}}^{r}\left\{\mathrm{e}^{-\int_{0}^{t} q(B(s)) \mathrm{d} s} f(B(t))\right\} .
$$

Clearly, $\mathrm{T}_{t}$ is an integral operator with kernel $h_{t}$ given by

$$
h_{t}(r, s)=\sum \mathrm{e}^{-\mathrm{e}_{k} t} \varphi_{k}(r) \varphi_{k}(s)
$$

2.2. $n$ non-colliding particles. Let us fix $n \in \mathbb{N}$ and define

$$
\mathbb{A}_{n}^{+}=\left\{\underline{r} \in \mathbb{R}^{n}: 0<r_{1}<\cdots<r_{n}\right\} .
$$

Let $\mathrm{L}_{i}$ be a copy of $\mathrm{L}$ acting on the $i$-th variable. Consider the closed self-adjoint extension of

$$
\sum_{1}^{n} \mathrm{~L}_{i}=\sum_{1}^{n}\left(\frac{1}{2} \frac{\partial^{2}}{\partial r_{i}^{2}}-q\left(r_{i}\right)\right)
$$

from $\mathrm{C}_{0}\left(\mathbb{A}_{n}^{+}\right)$and let $\mathrm{T}_{t}^{+}$be the corresponding contraction semigroup on $\mathbb{L}_{2}\left(\mathbb{A}_{n}^{+}\right)$.

In probabilistic terms, $\mathrm{T}_{t}^{+}$can be described as follows: For an $n$-tuple $\underline{r} \in \mathbb{A}_{n}^{+}$, set

$$
\hat{\mathbf{E}}^{r}=\hat{\mathbf{E}}^{r_{1}} \otimes \hat{\mathbf{E}}^{r_{2}} \otimes \cdots \otimes \hat{\mathbf{E}}^{r_{n}}
$$

Let $\underline{B}$ be the $n$-dimensional Brownian motion, and define

$$
\tau=\min \left\{t: \underline{B}(t) \notin \mathbb{A}_{n}^{+}\right\} .
$$

In other words, $\tau$ is the minimum between the first collision time and the first time the bottom trajectory exits from the positive semi-axis. Then,

$$
\mathrm{T}_{t}^{+} f(\underline{r})=\hat{\mathbf{E}}_{\underline{r}}\left\{\mathrm{e}^{-\sum_{i} \int_{0}^{t} q\left(B_{i}(s)\right) \mathrm{d} s} f(\underline{B}(t)) \mathbb{1}_{\tau>t}\right\} .
$$

$\mathrm{T}_{t}^{+}$is an integral operator on $\mathbb{L}_{2}\left(\mathbb{A}_{n}^{+}\right)$and, by the Karlin-McGregor formula, its kernel $\kappa_{t}$ is given by

$$
\kappa_{t}(\underline{r}, \underline{s})=\operatorname{det}\left\{h_{t}\left(r_{i}, s_{j}\right)\right\}
$$




\subsection{Limiting behaviour. Let}

$$
\Delta(\underline{r})=\operatorname{det}\left[\begin{array}{cccc}
\varphi_{1}\left(r_{1}\right) & \varphi_{2}\left(r_{1}\right) & \cdots & \varphi_{n}\left(r_{1}\right) \\
\varphi_{1}\left(r_{2}\right) & \varphi_{2}\left(r_{2}\right) & \cdots & \varphi_{n}\left(r_{2}\right) \\
\vdots & \vdots & \ddots & \vdots \\
\varphi_{1}\left(r_{n}\right) & \varphi_{2}\left(r_{n}\right) & \cdots & \varphi_{n}\left(r_{n}\right)
\end{array}\right]
$$

Note that $\Delta \in \mathbb{L}_{2}\left(\mathbb{A}_{n}^{+}\right)$.

Theorem 2.1. Set $\mathrm{D}_{n}=\sum_{1}^{n} \mathrm{e}_{\ell}$. Then,

$$
\lim _{t \rightarrow \infty} \mathrm{e}^{\mathrm{D}_{n} t} \kappa_{t}(\underline{r}, \underline{s})=\Delta(\underline{r}) \Delta(\underline{s}) .
$$

Moreover, for any $f \in \mathbb{L}_{2}\left(\mathbb{A}_{n}^{+}\right)$and for any $t>0$,

$$
\begin{gathered}
\lim _{T \rightarrow \infty} \frac{\mathbb{E}_{\underline{r}}\left\{\mathrm{e}^{-\sum_{i} \int_{0}^{T} q\left(B_{i}(s)\right) \mathrm{d} s} f(\underline{X}(t)) \mathbb{1}_{T<\tau_{+}}\right\}}{\mathbb{E}_{\underline{r}}\left\{\mathrm{e}^{-\sum_{i} \int_{0}^{T} q\left(B_{i}(s)\right) \mathrm{d} s} \mathbb{1}_{T<\tau_{+}}\right\}} \\
=\frac{\mathrm{e}^{\mathrm{D}_{n} t}}{\Delta(\underline{r})} \mathbb{E}_{\underline{r}}\left\{\mathrm{e}^{-\sum_{i} \int_{0}^{t} q\left(B_{i}(s)\right) \mathrm{d} s} f(\underline{B}(t)) \Delta(\underline{B}(t)) \mathbb{1}_{t<\tau_{+}}\right\} \\
=\frac{\mathrm{e}^{\mathrm{D}_{n} t}}{\Delta(\underline{r})} \mathrm{T}_{t}^{+}(f \Delta)(\underline{r}) \triangleq \mathrm{S}_{t}^{+} f(\underline{r}) .
\end{gathered}
$$

In its turn, $\mathrm{S}_{t}^{+}$is a diffusion semigroup with transition kernel

$$
q_{t}(\underline{r}, \underline{s})=\frac{\mathrm{e}^{\mathrm{D}_{n} t}}{\Delta(\underline{r})} \kappa_{t}(\underline{r}, \underline{s}) \Delta(\underline{s})
$$

which is self-adjoint on $\mathbb{L}_{2}\left(\mathbb{A}_{n}^{+}, \Delta^{2}\right)$; the generator of the corresponding ergodic diffusion on $\mathbb{A}_{n}^{+}$is given by

$$
\mathfrak{G}_{n}^{+}=\frac{1}{2} \sum_{1}^{n} \frac{\partial^{2}}{\partial r_{i}^{2}}+\nabla \log (\Delta)(\underline{r}) \cdot \nabla=\frac{1}{2 \Delta^{2}(\underline{r})} \operatorname{div}\left(\Delta^{2}(\underline{r}) \nabla\right) .
$$

Proof. Let us introduce the column vectors

$$
b_{\ell}=\left[\begin{array}{c}
\varphi_{\ell}\left(r_{1}\right) \\
\varphi_{\ell}\left(r_{2}\right) \\
\vdots \\
\varphi_{\ell}\left(r_{n}\right)
\end{array}\right] \quad(\ell=1, \ldots, n)
$$


and the volume form $F\left(c_{1}, \ldots, c_{n}\right)=\operatorname{det}\left[c_{1}, \ldots, c_{n}\right]$. Under our assumptions, (2.3), (2.7) and (2.12) imply, asymptotically as $t \rightarrow \infty$, that

$$
\begin{aligned}
\mathrm{e}^{\mathrm{D}_{n} t} \kappa_{t}(\underline{r}, \underline{s})(1+\mathrm{o}(1)) & =F\left(\sum_{1}^{n} \varphi_{\ell}\left(s_{1}\right) b_{\ell}, \sum_{1}^{n} \varphi_{\ell}\left(s_{2}\right) b_{\ell}, \ldots, \sum_{1}^{n} \varphi_{\ell}\left(s_{n}\right) b_{\ell}\right) \\
& =F\left(b_{1}, \ldots, b_{n}\right) \sum_{\sigma}(-1)^{\operatorname{sgn}(\sigma)} \prod_{\ell=1}^{n} \varphi_{\sigma_{\ell}}\left(s_{\ell}\right),
\end{aligned}
$$

and the first claim (2.14) follows. Above, $\sigma$ runs over all permutations of $\{1, \ldots, n\}$ and $\operatorname{sgn}(\sigma)= \pm 1$ denotes the signature of $\sigma$.

Modulo some technicalities, (2.15) follows from (2.14) and the Markov property.

Finally, $\mathfrak{G}_{n}$ in (2.17) is the generator of $\mathrm{S}_{t}^{+}$, since the generator of $\mathrm{T}_{t}^{+}$is the closed self-adjoint extension of $(2.9)$ from $\mathrm{C}_{0}\left(\mathbb{A}_{n}^{+}\right)$and since, by direct computation,

$$
\sum_{1}^{n}\left(\frac{1}{2} \frac{\partial^{2}}{\partial r_{i}^{2}}-q\left(r_{i}\right)\right) \Delta(\underline{r})=-\mathrm{D}_{n} \Delta(\underline{r}) .
$$

2.4. Dyson diffusions for Sturm-Liouville operators. For every $n \in \mathbb{N}$, the diffusion

$$
\mathrm{d} \underline{x}(t)=\mathrm{d} \underline{B}(t)+\nabla \log (\Delta)(\underline{x}(t)) \mathrm{d} t,
$$

with the generator $\mathfrak{G}_{n}$ described in Theorem 2.1, lives on $\mathbb{A}_{n}^{+}$and is reversible with respect to $\Delta^{2}(\underline{r}) \mathrm{d} \underline{r}$. In the sequel, we shall use $\mathbb{P}_{n}^{+}$for its distribution on $\mathrm{C}\left((-\infty,+\infty), \mathbb{A}_{n}^{+}\right)$and $\overline{\mathbb{P}}_{n}^{+; T}$ for the restriction of this distribution to $\mathrm{C}\left([-T, T], \mathbb{A}_{n}^{+}\right)$. Without loss of generality, let us assume that $\Delta^{2}(\underline{r})$ is a probability density (on $\mathbb{A}_{n}^{+}$). Note that the latter has a determinantal structure:

$$
\Delta^{2}(\underline{r})=\operatorname{det}\left\{\mathrm{K}_{n}\left(r_{i}, r_{j}\right)\right\}
$$

where the kernel $K_{n}$ is given by

$$
\mathrm{K}_{n}(r, s)=\sum_{\ell=1}^{n} \varphi_{\ell}(r) \varphi_{l}(s)
$$

In particular, the level density distribution is given by

$$
\rho_{n}(r)=\frac{1}{n} K_{n}(r, r)=\frac{1}{n} \sum_{1}^{n} \varphi_{\ell}^{2}(r) .
$$

There are similar determinantal formulas for level spacing, gap probabilities, etc. We note that the unpublished work [2] contains results on the universality of scaling limits (as $n \rightarrow \infty$ ) in this general Sturm-Liouville context. 


\section{Ordered Walks With area tilts.}

3.1. Underlying random walks and ordering of trajectories. The setup follows [15].

Let $p_{y}$ be an irreducible random walk kernel on $\mathbb{Z}$. The probability of a finite trajectory $\mathbb{X}=\left(X_{1}, X_{2}, \ldots, X_{k}\right)$ is $\mathrm{p}(\mathbb{X})=\prod_{i} p_{X_{i+1}-X_{i}}$. The product probability of $n$ finite trajectories $\underline{\mathbb{X}}=\left(\mathbb{X}^{1}, \ldots, \mathbb{X}^{n}\right)$ is

$$
\mathbf{P}(\underline{\mathbb{X}})=\prod_{\ell=1}^{n} \mathrm{p}\left(\mathbb{X}^{\ell}\right)
$$

Assumptions on p. Assume that

$$
\sum_{z \in \mathbb{Z}} z p_{z}=0 \text { and } \mathrm{p} \text { has finite exponential moments. }
$$

In the sequel, we, in order to facilitate the notation, shall assume that the variance satisfies,

$$
\sigma^{2}=\sum_{z \in \mathbb{Z}} z^{2} p_{z}=1
$$

Sets of trajectories. Let $u, v \in \mathbb{N}$. As in [15], $\mathcal{P}_{M, N,+}^{u, v}$ is used to denote the set of trajectories $\mathbb{X}$ starting at $u$ at time $M$, ending at $v$ at time $N$ and staying positive during the time interval $\{M, \ldots, N\}$.

Let $\underline{u}, \underline{v} \in \mathbb{N}^{n} \cap \mathbb{A}_{n}^{+}$and $M, N \in \mathbb{Z}$ with $M \leq N$. Let $\mathcal{P} \underline{u, \underline{v}}, N,+$ be the family of $n$ trajectories $\underline{\mathbb{X}}$ starting at $\underline{u}$ at time $M$, ending at $\underline{v}$ at time $N$ and satisfying

$$
0<X_{j}^{1}<X_{j}^{2}<\cdots<X_{j}^{n} \quad \forall j \in\{M+1, \ldots, N-1\} .
$$

For $\underline{u}, \underline{v} \in \mathbb{N}^{n}$, let $\mathcal{A}_{M, N,+}^{\underline{u}, \underline{v}}$ be the set of $n$ trajectories $\underline{\mathbb{X}}$ starting at $\underline{u}$ at time $M$, ending at $\underline{v}$ at time $N$, staying positive during the time interval $\{M, \ldots, N\}$ and satisfying

$$
X_{j}^{\ell} \neq X_{j}^{k} \quad \forall j \in\{M, \ldots, N\} \text { and } \ell \neq k .
$$

For $N>0$, we shall use the shorthand notations $\mathcal{P}_{N,+}^{u, v}=\mathcal{P}_{-N, N,+}^{\underline{u}, \underline{v}}$ and $\hat{\mathcal{P}}_{N, \underline{u}}^{\underline{u}, \underline{v}}=$ $\mathcal{P}_{1, N,+}^{\underline{u}, \underline{v}}$. The same convention applies for the shorthand notations $\mathcal{A}_{N,+}^{\underline{u}, \underline{v}}$ and $\hat{\mathcal{A}}_{N,+}^{u, v}$.

The model which we define below is a polymer measure over ordered trajectories $\mathcal{P}_{N,+}^{\underline{u}, \underline{v}}$. The families $\mathcal{A}_{M, N,+}^{\underline{u}, \underline{v}}$ are needed for an application of the Karlin-McGregor formula.

3.2. The model. Let $\left\{V_{\lambda}\right\}_{\lambda>0}$ be a family of self-potentials $V_{\lambda}: \mathbb{N} \rightarrow \mathbb{R}_{+}$. For a finite trajectory $\mathbb{X}=\left(X_{M}, \ldots, X_{N}\right)$, let $\mathrm{p}(\mathbb{X})=\prod_{i=M+1}^{N} \mathrm{p}\left(X_{i}-X_{i-1}\right)$ be its probability for the underlying random walk, and let us introduce the tilted weights

$$
\mathrm{w}_{\lambda}(\mathbb{X})=\mathrm{e}^{-\sum_{M+1}^{N} V_{\lambda}\left(X_{i}\right)} \mathrm{p}(\mathbb{X}) .
$$


Given $u, v \in \mathbb{N}$ and $\lambda>0$, define the partition functions and the probability distributions

$$
Z_{N,+, \lambda}^{u, v}=\sum_{\mathbb{X} \in \mathcal{P}_{N,+}^{u, v}} \mathrm{w}_{\lambda}(\mathbb{X}) \text { and } \mathbb{P}_{N,+, \lambda}^{u, v}(\mathbb{X})=\frac{1}{Z_{N,+, \lambda}^{u v}} \mathrm{w}_{\lambda}(\mathbb{X}) \mathbb{1}_{\left\{\mathbb{X} \in \mathcal{P}_{N,+}^{u, v}\right\}}
$$

In the case of an $n$-tuple $\underline{\mathbb{X}}=\left(\mathbb{X}^{1}, \ldots, \mathbb{X}^{n}\right)$ of trajectories, we consider the product weights $\mathrm{w}_{\lambda}(\underline{\mathbb{X}})=\prod_{i=1}^{n} \mathrm{w}_{\lambda}\left(\mathbb{X}^{i}\right)$. If $\mathcal{S}$ is a finite or countable set of such tuples, then the corresponding restricted partition functions are denoted by

$$
Z_{\lambda}[\mathcal{S}]=\sum_{\underline{\mathbb{X}} \in \mathcal{S}} \mathrm{w}_{\lambda}(\underline{\mathbb{X}})
$$

We shall use the shorthand notations $Z_{N,+, \lambda}^{\underline{u}, \underline{v}}=Z_{\lambda}\left[\mathcal{P}_{N,+}^{\underline{u}, \underline{v}}\right]$ and $\hat{Z}_{N,+, \lambda}^{\underline{u}, \underline{v}}=Z_{\lambda}\left[\hat{\mathcal{P}}_{N,+}^{\underline{u}, \underline{v}}\right]$. Finally, let us define the probability distribution $\mathbb{P}_{N, \underline{u},+, \lambda}$ on $\mathcal{P}_{N,+}^{\underline{u}, \underline{v}}$ by

$$
\mathbb{P} \frac{\underline{u}, \underline{v}}{N,+, \lambda}(\underline{\mathbb{X}})=\frac{1}{Z_{N,+, \lambda}^{\underline{u}, \underline{v}}} \mathrm{w}_{\lambda}(\underline{\mathbb{X}}) \mathbb{1}_{\left\{\underline{\mathbb{X}} \in \mathcal{P} \frac{u, v}{N,+}\right\}}
$$

The term $\sum_{-N+1}^{N} V_{\lambda}\left(X_{i}\right)$ represents a generalized (non-linear) area below the trajectory $\mathbb{X}$. It reduces to (a multiple of) the usual area when $V_{\lambda}(x)=\lambda x$. As in [15], we make the following set of assumptions on $V_{\lambda}$ :

3.3. Assumptions on $V_{\lambda}$ and the scale $H_{\lambda}$. For any $\lambda>0$, the function $V_{\lambda}$ on $[0, \infty)$ is continuous, monotone increasing and satisfies

$$
V_{\lambda}(0)=0 \quad \text { and } \quad \lim _{x \rightarrow \infty} V_{\lambda}(x)=\infty .
$$

In particular, the relation

$$
H_{\lambda}^{2} V_{\lambda}\left(H_{\lambda}\right)=1
$$

determines unambiguously the quantity $H_{\lambda}$. Furthermore, we make the assumptions that $\lim _{\lambda \downarrow 0} H_{\lambda}=\infty$ and that there exists a function $q \in \mathrm{C}^{2}\left(\mathbb{R}^{+}\right)$such that

$$
\lim _{\lambda \downarrow 0} H_{\lambda}^{2} V_{\lambda}\left(r H_{\lambda}\right)=q(r),
$$

uniformly on compact subsets of $\mathbb{R}_{+}$. Note that $H_{\lambda}$, respectively $H_{\lambda}^{2}$, plays the role of the spatial, respectively temporal, scale in the invariance principle which is formulated below in Theorem A.

Furthermore, we shall assume that there exist $\lambda_{0}>0$ and a (continuous nondecreasing) function $q_{0} \geq 0$ with $\lim _{r \rightarrow \infty} q_{0}(r)=\infty$ such that, for all $\lambda \leq \lambda_{0}$,

$$
H_{\lambda}^{2} V_{\lambda}\left(r H_{\lambda}\right) \geq q_{0}(r) \text { on } \mathbb{R}_{+} \text {. }
$$

Finally, we assume that $q_{0}$ grows to $\infty$ sufficiently fast; namely, for any $\kappa>0$,

$$
\int_{0}^{\infty} \mathrm{e}^{-\kappa q_{0}(r)} \mathrm{d} r<\infty
$$


Presumably, our main results hold without assumption (3.14). However, since it is rather soft and since it implies the claim of the technically very convenient Lemma 4.2 below, we decided to keep it.

Remark 1. A natural class of examples of family of potentials satisfying assumptions (3.10)-(3.14) is given by $V_{\lambda}(x)=\lambda x^{\alpha}$ with $\alpha>0$. For the latter, $H_{\lambda}=$ $\lambda^{-1 /(2+\alpha)}$ and $q(r)=q_{0}(r)=r^{\alpha}$. In this way, the case of linear area tilts $\alpha=1$ corresponds to the familiar Airy rescaling $H_{\lambda}=\lambda^{-1 / 3}$.

3.4. The result. We set $h_{\lambda}=H_{\lambda}^{-1}$. The paths are rescaled as follows: For $t \in h_{\lambda}^{2} \mathbb{Z}$, define

$$
\underline{x}^{\lambda}(t)=h_{\lambda} \underline{X}_{H_{\lambda}^{2} t}=\frac{1}{H_{\lambda}} \underline{X}_{H_{\lambda}^{2} t}
$$

Then, extend $\underline{x}^{\lambda}$ to any $t \in \mathbb{R}$ by linear interpolation. In this way, given $T>0$ and $\underline{u}, \underline{v}$, we can talk about the induced distribution $\mathbb{P}_{\underline{N},+, \lambda}^{\underline{u}, T}$ on the space of continuous functions $\mathrm{C}\left([-T, T], \mathbb{A}_{n}^{+}\right)$.

Theorem A. Let $\lambda_{N}$ be a sequence satisfying

$$
\lim _{N \rightarrow \infty} \lambda_{N}=0 \quad \text { and } \quad \lim _{N \rightarrow \infty} a_{N} \triangleq \lim _{N \rightarrow \infty} \frac{N}{H_{\lambda_{N}}^{2}}=\infty .
$$

Fix any $C \in(0, \infty)$ and any $T>0$. Then, the sequence of distributions $\frac{\mathbb{u}, \underline{v} ; T}{N,+, \lambda_{N}}$ converges weakly to the distribution $\mathbb{P}_{n}^{+; T}$ of the ergodic diffusion $\underline{x}(\cdot)$ in $(2.20)$, uniformly in $v_{n}, u_{n} \leq C H_{\lambda_{N}}$.

3.5. Non-strict constraints. In the sequel, we shall focus on the strict constraints expressed in (3.4). However, a rather straightforward modification of our arguments would imply that the conclusion still holds when the ordering in (3.4) is non-strict, that is, when we instead require that

$$
0 \leq X_{j}^{1} \leq X_{j}^{2} \leq \ldots \leq X_{j}^{n} \quad \forall j \in\{M, \ldots, N\}
$$

Namely, let $\mathcal{P} \underline{\underline{u}, \underline{v}}, N, 0$ be the family of $n$ trajectories $\underline{\mathbb{X}}$ starting at $\underline{u}$ at time $M$, ending at $\underline{v}$ at time $N$ and satisfying (3.17). As in the case of strict ordering, we use abbreviation $\mathcal{P}_{N, 0}^{\underline{u}, \underline{v}}=\mathcal{P}_{-N, \underline{u}, \underline{\underline{u}}, 0}$. Define (recall (3.1))

$$
Z_{N, 0, \lambda}^{\underline{u}, \underline{v}}=\sum_{\underline{\mathbb{X}} \in \mathcal{P} \frac{u, v}{N, 0}} \mathrm{e}^{-\sum_{\ell=1}^{n} \sum_{i=-N}^{N} V_{\lambda}\left(X_{i}^{\ell}\right)} \mathbf{P}(\underline{\mathbb{X}})
$$

and

$$
\mathbb{P} \frac{\underline{u}, \underline{v}}{N, 0, \lambda}(\underline{\mathbb{X}})=\frac{\mathrm{e}^{-\sum_{\ell=1}^{n} \sum_{i=-N}^{N} V_{\lambda}\left(X_{i}^{\ell}\right)} \mathbf{P}(\underline{\mathbb{X}})}{Z_{N}^{\underline{u}, \underline{v}, 0}, \lambda} \mathbb{1}_{\left\{\underline{\mathbb{X}} \in \mathcal{P} \frac{\underline{u}, \underline{v}}{N, 0}\right\}} .
$$

Corollary 3.1. Under the same assumptions, the conclusions of Theorem A hold for the family of measures $\mathbb{P} \underline{\underline{u}, \underline{v}}, 0, \lambda$. 
3.6. Rescaling and the corresponding notation. It will be convenient to adjust our notations to the running scales $h_{\lambda}$. Define:

$$
\mathbb{N}_{\lambda}=h_{\lambda} \mathbb{N}, \quad \mathbb{A}_{n, \lambda}^{+}=\mathbb{A}_{n}^{+} \cap\left(\mathbb{N}_{\lambda}\right)^{n} \quad \text { and } \quad \mathbb{Z}_{\lambda}=h_{\lambda}^{2} \mathbb{Z}
$$

In this way, $\underline{x}^{\lambda}(t)$ in (3.15) belongs to $\mathbb{A}_{n, \lambda}^{+}$for every $t \in \mathbb{Z}_{\lambda}$.

For $a, b, t \in \mathbb{Z}_{\lambda}$ and $\underline{r}, \underline{s} \in \mathbb{A}_{n, \lambda}^{+}$, we shall write, with a slight abuse of notation,

$$
\mathcal{P}_{a, b,+, \lambda}^{\underline{r}, \underline{s}} \equiv \mathcal{P}_{H_{\lambda}^{2} a, H_{\lambda}^{2} b,+, \lambda}^{H_{\underline{r}} \underline{r}, H_{\lambda} \underline{s}} \text { and similarly for } \mathcal{P}_{t,+, \lambda}^{\underline{r}, \underline{s}}, \hat{\mathcal{P}}_{t,+, \lambda}^{\underline{r}, \underline{s}} \mathcal{A}_{t,+, \lambda}^{\underline{r}, \underline{s}} \text { and } \hat{\mathcal{A}}_{t,+, \lambda}^{\underline{r}, \underline{s}} \text {. }
$$

The same conventions apply to partition functions (e.g., we shall write $\hat{Z}_{t,+, \lambda}^{\underline{r}, \underline{s}}=$ $\left.Z_{\lambda}\left[\hat{\mathcal{P}}_{t,+, \lambda}^{\underline{r}, \underline{s}}\right]\right)$ and for probability distributions (e.g., we shall write $\mathbb{P}_{a,+, \lambda}^{\underline{r}, \underline{s}}$ for $a \in \mathbb{Z}_{\lambda}$ and $\left.\underline{r}, \underline{s}, \in \mathbb{A}_{n, \lambda}^{+}\right)$.

With the above notations, Theorem A can be restated as follows: Let

$$
\lim _{N \rightarrow \infty} \lambda_{N}=0 \text { and } \lim _{N \rightarrow \infty} a_{N}=\infty .
$$

Then, the family of distributions $\mathbb{P}_{a_{N}, \underline{r} ;+, \lambda_{N}}^{\underline{r}}$ converges weakly to the distribution $\mathbb{P}_{n}^{+; T}$ of the ergodic diffusion $\underline{x}(\cdot)$, uniformly in $r_{n}, s_{n} \leq C$.

Our proofs rely on the properties of the underlying rescaled random walks (without area tilts). The corresponding notation for the latter follows the above convention adopted for polymer measures: Given $\lambda>0$ and $\underline{r} \in \mathbb{N}_{\lambda}^{n}$, we use $\hat{\mathbf{P}}_{\lambda}^{r}$ for the law of the rescaled walk started at time zero at $\underline{r}$. The restriction of $\hat{\mathbf{P}}_{\lambda}^{\frac{r}{\lambda}}$ to the set of trajectories which stay in $\mathbb{A}_{n, \lambda}^{+}$during the interval $[0, t]$ is denoted by $\hat{\mathbf{P}}_{t,+, \lambda}^{r}$. When the end-point $t$ is clear from the context, we will sometimes use the shorthand notation $\hat{\mathbf{P}}_{+, \lambda}^{r}$. Finally, given $\underline{s} \in \mathbb{N}_{\lambda}^{n}$ and $t \in \mathbb{Z}_{\lambda}$, we use

$$
\hat{\mathbf{P}}_{t, \lambda}^{r, s}=\hat{\mathbf{P}}_{\lambda}^{r}\left(\cdot \mid \underline{x}^{\lambda}(t)=\underline{s}\right) \quad \text { and } \quad \hat{\mathbf{P}}_{t,+, \lambda}^{r, s}=\hat{\mathbf{P}}_{+, \lambda}^{r}\left(\cdot \mid \underline{x}^{\lambda}(t)=\underline{s}\right) .
$$

3.7. Structure of the argument. As $\lambda \downarrow 0$, the following notion of convergence is employed: Consider the spaces $\ell_{2}\left(\mathbb{N}_{\lambda}\right)$ and $\ell_{2}\left(\mathbb{A}_{n, \lambda}^{+}\right)$with scalar products

$$
\langle f, g\rangle_{2, \lambda}=h_{\lambda} \sum_{r \in \mathbb{N}_{\lambda}} f(r) g(r) \text { and, respectively, }\langle f, g\rangle_{2, \lambda}=h_{\lambda}^{n} \sum_{\underline{r} \in \mathbb{A}_{n, \lambda}^{+}} f(\underline{r}) g(\underline{r}) .
$$

Let $\rho_{\lambda}: \mathbb{L}_{2}\left(\mathbb{R}_{+}\right) \rightarrow \ell_{2}\left(\mathbb{N}_{\lambda}\right)$ and $\rho_{\lambda, n}: \mathbb{L}_{2}\left(\mathbb{A}_{n}^{+}\right) \rightarrow \ell_{2}\left(\mathbb{A}_{n, \lambda}^{+}\right)$be linear contractions; for instance, to fix the ideas, set

$$
\rho_{\lambda} u(r)=\frac{1}{h_{\lambda}} \int_{\left(r-h_{\lambda}\right)_{+}}^{r} u(s) \mathrm{d} s \text { and } \rho_{\lambda, n} u(\underline{r})=\frac{1}{h_{\lambda}^{n}} \int_{\left(r_{1}-h_{\lambda}\right)_{+}}^{r_{1}} \ldots \int_{\left(r_{n}-h_{\lambda}\right)_{+}}^{r_{n}} u(\underline{s}) \mathbb{1}_{\left\{\underline{s} \in \mathbb{A}_{n}^{+}\right\}} \mathrm{d} \underline{s} .
$$

Above, $s_{+}=s \vee 0$ for any $s \in \mathbb{R}$. Let us say that a sequence $u_{\lambda} \in \ell_{2}\left(\mathbb{N}_{\lambda}\right)$ converges to $u \in \mathbb{L}_{2}\left(\mathbb{R}_{+}\right)$, which we denote $u=\lim u_{\lambda}$, if

$$
\lim _{\lambda \downarrow 0}\left\|u_{\lambda}-\rho_{\lambda} u\right\|_{2, \lambda}=0 .
$$


The same definition applies for sequences $u_{\lambda} \in \ell_{2}\left(\mathbb{A}_{n, \lambda}^{+}\right)$and, accordingly, for the limiting $u$ in $\mathbb{L}_{2}\left(\mathbb{A}_{n}^{+}\right)$. Note that, in both cases, if $u=\lim _{\lambda \downarrow 0} u_{\lambda}$ and $v=\lim _{\lambda \downarrow 0} v_{\lambda}$, then

$$
\lim _{\lambda \downarrow 0}\left\langle u_{\lambda}, v_{\lambda}\right\rangle_{2, \lambda}=\int_{0}^{\infty} u(r) v(r) \mathrm{d} r, \text { respectively, } \lim _{\lambda \downarrow 0}\left\langle u_{\lambda}, v_{\lambda}\right\rangle_{2, \lambda}=\int_{\mathbb{A}_{n}^{+}} u(\underline{r}) v(\underline{r}) \mathrm{d} \underline{r} .
$$

STEP 1. (Convergence of one-dimensional and product semi-groups.) Recall that $\mathrm{T}_{t}$ is an integral operator whose kernel $h_{t}$ is defined in (2.7). [15, Proposition 3] implies that if a sequence $f_{\lambda} \in \ell_{2}\left(\mathbb{N}_{\lambda}\right)$ converges to $f \in \mathbb{L}_{2}\left(\mathbb{R}_{+}\right)$, then, for any $t>0$,

$$
\lim _{\lambda \downarrow 0} \sum_{s \in \mathbb{N}_{\lambda}} \hat{Z}_{t,+, \lambda}^{r, s} f_{\lambda}(s)=\int_{0}^{\infty} h_{t}(r, s) f(s) \mathrm{d} s
$$

in the sense of (3.24) above. In particular, for any $f, g \in \mathrm{C}_{0}\left(\mathbb{R}_{+}\right)$,

$$
\lim _{\lambda \downarrow 0} h_{\lambda} \sum_{r \in \mathbb{N}_{\lambda}} \sum_{s \in \mathbb{N}_{\lambda}} g(r) \hat{Z}_{t,+, \lambda}^{r, s} f(s)=\int_{0}^{\infty} \int_{0}^{\infty} g(r) h_{t}(r, s) f(s) \mathrm{d} r \mathrm{~d} s .
$$

We claim:

Proposition 3.1. Assume that the sequence $f_{\lambda} \in \ell_{2}\left(\mathbb{A}_{n, \lambda}^{+}\right)$converges to $f \in C_{0}\left(\mathbb{A}_{n}^{+}\right)$. Let $\sigma$ be a permutation of $\{1, \ldots, n\}$. Then, for any $t>0$,

$$
\lim _{\lambda \downarrow 0} \sum_{\underline{s} \in \mathbb{A}_{n, \lambda}^{+}} \prod_{i=1}^{n} \hat{Z}_{t,+, \lambda}^{r_{i}, s_{\sigma_{i}}} f_{\lambda}(\underline{s})=\int_{\mathbb{A}_{n}^{+}} \prod_{i=1}^{n} h_{t}\left(r_{i}, s_{\sigma_{i}}\right) f(\underline{s}) \mathrm{d} \underline{s},
$$

in the sense of (3.24) above. In particular, let $f, g \in \mathrm{C}_{0}\left(\mathbb{A}_{n}^{+}\right)$. Then, for any $t>0$,

$$
\lim _{\lambda \downarrow 0} h_{\lambda}^{n} \sum_{\underline{r} \in \mathbb{A}_{n, \lambda}^{+}} \sum_{\underline{s} \in \mathbb{A}_{n, \lambda}^{+}} g(\underline{r}) \prod_{i=1}^{n} \hat{Z}_{t,+, \lambda}^{r_{i}, s_{\sigma_{i}}} f(\underline{s})=\int_{\mathbb{A}_{n}^{+}} \int_{\mathbb{A}_{n}^{+}} g(\underline{r}) \prod_{i=1}^{n} h_{t}\left(r_{i}, s_{\sigma_{i}}\right) f(\underline{s}) \mathrm{d} \underline{r} \mathrm{~d} \underline{s} .
$$

STEP 2. (Karlin-McGregor formula and probabilistic estimates.) Let $\underline{r}, \underline{s} \in \mathbb{A}_{n, \lambda}^{+}$. By an application of Karlin-McGregor formula (see [17, Section 5]),

$$
\operatorname{det}\left\{\hat{Z}_{t,+, \lambda}^{r_{i}, s_{j}}\right\}=\sum_{\sigma}(-1)^{\operatorname{sgn}(\sigma)} Z\left[\hat{\mathcal{A}}_{t,+, \lambda}^{r, \underline{s}_{\sigma}}\right] .
$$

Above, $\left(\underline{s}_{\sigma}\right)_{i} \equiv s_{\sigma_{i}}$.

Recall our notation for rescaled norms: $\left\|f_{\lambda}\right\|_{2, \lambda}^{2}=h_{\lambda}^{n} \sum_{\underline{r}} f_{\lambda}^{2}(\underline{r})$. We claim:

Theorem 3.1. (a) For any $t_{0}>0$ and for any non-trivial permutation $\sigma \neq \mathrm{Id}$,

$$
\lim _{\lambda \downarrow 0} \sum_{\underline{s} \in \mathbb{A}_{n, \lambda}^{+}} Z_{\lambda}\left[\hat{\mathcal{A}}_{t,+, \lambda}^{\underline{r}, \underline{s} \sigma}\right] f_{\lambda}(\underline{s})=0
$$


in the sense of (3.24), uniformly in $t \geq t_{0}$ and in $\left\|f_{\lambda}\right\|_{2, \lambda}=1$.

(b) For any $t_{0}>0$,

$$
\lim _{\lambda \downarrow 0} \sum_{\underline{s} \in \mathbb{A}_{n, \lambda}^{+}}\left(Z_{\lambda}\left[\hat{\mathcal{A}}_{t,+, \lambda}^{\underline{r}, \underline{s}}\right]-Z_{\lambda}\left[\hat{\mathcal{P}}_{t,+, \lambda}^{\underline{r}, \underline{s}}\right]\right) f_{\lambda}(\underline{s})=0,
$$

as well, also uniformly in $t \geq t_{0}$ and $\left\|f_{\lambda}\right\|_{2, \lambda}=1$.

Recall our notation $\hat{Z}_{t,+, \lambda}^{\underline{r}, \underline{s}}=Z_{\lambda}\left[\hat{\mathcal{P}}_{t,+,+,}^{\underline{r}, \underline{s}}\right]$ and $\kappa_{t}(\underline{r}, \underline{s})=\operatorname{det}\left\{h_{t}\left(r_{i}, s_{j}\right)\right\}$. Proposition 3.1 and Theorem 3.1 imply:

Theorem 3.2. For any $t>0$ and any sequence $f_{\lambda} \in \ell_{2}\left(\mathbb{A}_{n, \lambda}^{+}\right)$with $\lim _{\lambda \downarrow 0} f_{\lambda}=f$,

$$
\lim _{\lambda \downarrow 0} \sum_{\underline{s} \in \mathbb{A}_{n, \lambda}^{+}} \hat{Z}_{t,+, \lambda}^{\underline{r}, \underline{s}} f_{\lambda}(\underline{s})=\int_{\mathbb{A}_{n}^{+}} \kappa_{t}(\underline{r}, \underline{s}) f(\underline{s}) \mathrm{d} \underline{s} .
$$

In particular, for any $f, g \in \mathrm{C}_{0}\left(\mathbb{A}_{n}^{+}\right)$,

$$
\lim _{\lambda \downarrow 0} h_{\lambda}^{n} \sum_{\underline{r} \in \mathbb{A}_{n, \lambda}^{+}} \sum_{\underline{s} \in \mathbb{A}_{n, \lambda}^{+}} g(\underline{r}) Z_{t,+, \lambda}^{\underline{r}, \underline{s}} f(\underline{s})=\int_{\mathbb{A}_{n}^{+}} \int_{\mathbb{A}_{n}^{+}} g(\underline{r}) \kappa_{t}(\underline{r}, \underline{s}) f(\underline{s}) \mathrm{d} \underline{r} \mathrm{~d} \underline{s} .
$$

STEP 3. (Tightness.) We claim:

Proposition 3.2. Fix any $T>0$. Under the conditions of Theorem A, the family $\left\{\mathbb{P}_{a_{N}, \underline{s} ;,+, \lambda_{N}}\right\}$ of probability distributions on $\mathrm{C}\left([-T, T], \mathbb{A}_{n}^{+}\right)$is tight.

STEP 4. (Mixing.) We claim:

Theorem 3.3. For any $C<\infty$, there exist $c_{1}, c_{2}>0$ such that, for any $K>0$,

$$
\left\|\mathbb{P}_{a,+, i, \lambda}^{\underline{r} ; T}-\mathbb{P} \frac{\underline{w}, \underline{z} ; T}{b,+, \lambda}\right\|_{\mathrm{var}} \leq c_{1} \mathrm{e}^{-c_{2} K}
$$

holds uniformly in $\lambda$ small, $a, b \in \mathbb{Z}_{\lambda}$ with $a, b \geq(K+T)$ and uniformly in $\underline{r}, \underline{s}, \underline{w}, \underline{z} \in$ $\mathbb{A}_{n, \lambda}^{+}$with $r_{n}, s_{n}, w_{n}, z_{n} \leq C$.

STEP 5. (Convergence of finite-dimensional distributions.) Fix $T>0$. Let $\lambda_{N} \downarrow 0$ and let $a_{N} \in \mathbb{Z}_{\lambda_{N}}$ satisfy $\lim a_{N}=\infty$. Let $f, g \in \mathrm{C}_{0}\left(\mathbb{A}_{n}^{+}\right)$be two non-negative and non-identically zero functions. For $M \in \mathbb{Z}_{\lambda}, M>T$, define the partition functions (rescaled as in (3.19))

$$
Z_{M,+, \lambda}^{g, f}=h_{\lambda}^{n} \sum_{\underline{r} \in \mathbb{A}_{n}^{+}} \sum_{\underline{s} \in \mathbb{A}_{n}^{+}} g(\underline{r}) Z_{M,+, \lambda}^{\underline{r}, \underline{s}} f(\underline{s})>0
$$

and let $\mathbb{P}_{M,+, \lambda}^{g, f ; T}$ be the corresponding induced probability distribution on $\mathrm{C}\left([-T, T], \mathbb{A}_{n}^{+}\right)$. By Theorem 3.3, under the conditions of Theorem A,

$$
\lim _{M \rightarrow \infty} \lim _{N \rightarrow \infty}\left\|\mathbb{P}_{a_{N}, \underline{r} ;, T}^{,+, \lambda_{N}}-\mathbb{P}_{M,+, \lambda_{N}}^{g, f ; T}\right\|_{\mathrm{var}}=0
$$

uniformly in $r_{n}, s_{n} \leq C$. 
Let now $-T \leq t_{1}<t_{2}<\ldots t_{m} \leq T$ and let $u_{1}, \ldots, u_{m} \in \mathrm{C}_{0}\left(\mathbb{A}_{n}^{+}\right)$. By Theorem 3.2 ,

$$
\begin{aligned}
& \lim _{\lambda \downarrow 0} \mathbb{E}_{M,+, \lambda}^{g, f ; T}\left(\prod_{i=1}^{m} u_{i}\left(\underline{x}^{\lambda}\left(t_{i}\right)\right)\right)= \\
& \frac{\int g(\underline{r}) \int \kappa_{t_{1}+M}\left(\underline{r}, \underline{r}^{1}\right) u_{1}\left(\underline{r}^{1}\right) \int \ldots u_{m}\left(\underline{r}^{m}\right) \int \kappa_{M-t_{m}}\left(\underline{r}^{m}, \underline{s}\right) f(\underline{s}) \mathrm{d} \underline{s} \mathrm{~d} \underline{r}^{m} \cdots \mathrm{d} \underline{r}}{\iint g(\underline{r}) \kappa_{2 M}(\underline{r}, \underline{s}) f(\underline{s}) \mathrm{d} \underline{s} \mathrm{~d} \underline{r}} .
\end{aligned}
$$

Above, all integrals are over $\mathbb{A}_{n}^{+}$. In view of (2.14) and by the definition of the semigroup $\mathrm{S}_{t}^{+}$in (2.15), the formulas (3.37) and (3.38) imply:

Proposition 3.3. Fix $T>0,-T \leq t_{1}<t_{2}<\ldots t_{m} \leq T$ and let $u_{1}, \ldots, u_{m}$ be bounded continuous functions on $\mathbb{A}_{n}^{+}$. Let $\lambda_{N}$ and $a_{N}$ satisfy the assumptions of Theorem A. Then,

$$
\begin{aligned}
& \lim _{N \rightarrow \infty} \mathbb{E}_{a_{N},+, \lambda_{N}}^{\underline{r}, \underline{s}}\left(\prod_{i=1}^{m} u_{i}\left(\underline{x}^{\lambda_{N}}\left(t_{i}\right)\right)\right)= \\
& \int \Delta^{2}\left(\underline{r}^{1}\right) u_{1}\left(\underline{r}^{1}\right) \int q_{t_{2}-t_{1}}\left(\underline{r}^{1}, \underline{r}^{2}\right) u_{2}\left(\underline{r}^{2}\right) \int \cdots \int q_{t_{m}-t_{m-1}}\left(\underline{r}^{m-1}, \underline{r}^{m}\right) \mathrm{d} \underline{r}^{m} \cdots \mathrm{d} \underline{r}^{1},
\end{aligned}
$$

uniformly in $r_{n}, s_{n} \leq C$. Above, $q_{t}$ is the transition kernel of $\mathrm{S}_{t}^{+}$, as defined in (2.16).

STEP 6. (Conclusion of the Proof.) By Proposition 3.2, the sequence of measures $\left\{\mathbb{P}_{a_{N}, \underline{r}, \underline{s} ; T}^{,+\lambda_{N}} ; r_{n}, s_{n} \leq C\right\}$ on $\mathrm{C}\left([-T, T], \mathbb{A}_{n}^{+}\right)$is tight for any $T>0$ fixed. By Proposition 3.3, its finite-dimensional distributions converge to the finite-dimensional distributions of the Dyson diffusion $\underline{x}(\cdot)$ in $(2.20)$.

3.8. Organization of the technical part of the paper. We still have to prove Theorem 3.1, Propositions 3.1 and 3.2 and Theorem 3.3. This will be done in Section 4, Section 5 and, respectively, in Sections 6 and 7. The proof of Theorem 3.3 is by far the most techically loaded part of the paper, and it relies on the probabilistic estimates (I.1)-(I.3), which are based on strong approximation techniques and on invariance principles for random walks in Weyl chambers. The derivation of (I.1)(I.3) is relegated to the Appendix.

\section{Proof of Theorem 3.1}

4.1. Preliminary estimates. Let us start with three preliminary estimates. The first one is just a rough local CLT estimate for the underlying random walk without area tilts: Recall that whenever we write quantities like $\hat{Z}_{t,+, \lambda}^{r, s}$, we are implicitly assuming that $t \in \mathbb{Z}_{\lambda}=h_{\lambda}^{2} \mathbb{Z}$ and that $r, s \in \mathbb{N}_{\lambda}=h_{\lambda} \mathbb{N}$.

Lemma 4.1. For any $t_{0}>0$, there exists a finite constant $c_{1}\left(t_{0}\right)$ such that

$$
\sup _{t \geq t_{0}} \sup _{r, s \in \mathbb{N}_{\lambda}} \hat{Z}_{t,+, \lambda}^{r, s} \leq c_{1}\left(t_{0}\right) h_{\lambda}
$$


for all $\lambda$ sufficiently small.

Indeed, since $V_{\lambda} \geq 0, Z_{t,+, \lambda}^{r, s} \leq \hat{\mathbf{P}}_{\lambda}^{r}\left(x^{\lambda}(t)=s\right)$.

Next, following [15], let us introduce

$$
\hat{Z}_{t,+, \lambda}^{r, \emptyset}=\sum_{s \in \mathbb{N}_{\lambda}} \hat{Z}_{t,+, \lambda}^{r, s} .
$$

Lemma 4.2. For any $t_{0}>0$, there exists a finite constant $c_{2}\left(t_{0}\right)$ such that

$$
\sup _{t \geq t_{0}} h_{\lambda} \sum_{r \in \mathbb{N}_{\lambda}} \hat{Z}_{t,+, \lambda}^{r, \emptyset} \leq c_{2}\left(t_{0}\right),
$$

for all $\lambda$ sufficiently small. Furthermore,

$$
\lim _{K \rightarrow \infty} \sup _{t \geq t_{0}} h_{\lambda} \sum_{\substack{r \in \mathbb{N}_{\lambda} \\ r \geq K}} \hat{Z}_{t,+, \lambda}^{r, \emptyset}=0,
$$

uniformly in $\lambda$ sufficiently small.

Proof. Note that Lemma 4.2 is in general wrong without the additional Assumption (3.14). On the other hand, under Assumption (3.13), it is straightforward to check that there exists $\kappa=\kappa\left(t_{0}\right)>0$ such that

$$
\sup _{t \geq t_{0}} \hat{Z}_{t,+, \lambda}^{r, \emptyset} \leq \mathrm{e}^{-\kappa \min \left\{q_{0}(r / 2), r^{2}\right\}}
$$

for all $\lambda$ small and all $r \in \mathbb{N}_{\lambda}$. Both (4.3) and (4.4) follow now from (3.14).

The third estimate is again on the underlying random walk, or more precisely on two independent copies $\left(x^{\lambda}, y^{\lambda}\right)$ of this walk. Namely,

Lemma 4.3. For any $\delta_{0} \in \mathbb{R}_{+}$and $K \in \mathbb{R}_{+}$fixed,

$$
\lim _{\lambda \rightarrow 0} \max _{\delta \geq \delta_{0}} \max _{0<u<v \leq K} \hat{\mathbf{P}}_{\lambda}^{u} \otimes \hat{\mathbf{P}}_{\lambda}^{v}\left(x^{\lambda}(\delta)>y^{\lambda}(\delta) ; x^{\lambda}(t) \neq y^{\lambda}(t) \forall t \in[0, \delta] \cap \mathbb{Z}_{\lambda}\right)=0 .
$$

Proof. The claim follows from [20, Theorem 1] and local limit asymptotics for random walks with exponential tails.

4.2. Proof of Theorem 3.1 (a). Pick $f_{\lambda} \in \ell_{2}\left(\mathbb{A}_{n, \lambda}^{+}\right)$with $\left\|f_{\lambda}\right\|_{2, \lambda}^{2}=1$. Set

$$
u_{\lambda}(\underline{r})=\sum_{\underline{s} \in \mathbb{A}_{n, \lambda}^{+}} Z_{\lambda}\left[\hat{\mathcal{A}}_{t,+, \lambda}^{\underline{r}, \underline{s}_{\sigma}}\right] f_{\lambda}(\underline{s}) .
$$

In order to prove (3.31), we need to check that, whenever $\sigma \neq \mathrm{Id}$,

$$
\lim _{\lambda \downarrow 0} h_{\lambda}^{n} \sum_{\underline{r} \in \mathbb{A}_{n, \lambda}^{+}} u_{\lambda}(\underline{r})^{2}=0,
$$


uniformly in $f$ with $\left\|f_{\lambda}\right\|_{2, \lambda}^{2}=1$. By the Cauchy-Schwarz inequality and Lemma 4.1,

$$
\begin{aligned}
u_{\lambda}(\underline{r})^{2}=\left(\sum_{\underline{s} \in \mathbb{A}_{n, \lambda}^{+}} Z_{\lambda}\left[\hat{\mathcal{A}}_{t,+, \lambda}^{r, \underline{s} \sigma}\right] f_{\lambda}(\underline{s})\right)^{2} & \leq\left(\sum_{\underline{s} \in \mathbb{A}_{n, \lambda}^{+}} Z\left[\hat{\mathcal{A}}_{t,+, \lambda}^{r, \underline{s}_{\sigma}}\right]\right)\left(\sum_{\underline{s} \in \mathbb{A}_{n, \lambda}^{+}} Z_{\lambda}\left[\hat{\mathcal{A}}_{t,+, \lambda}^{r, \underline{s}_{\sigma}}\right] f_{\lambda}^{2}(\underline{s})\right) \\
& \leq c_{1}\left(t_{0}\right)^{n} \sum_{\underline{s} \in \mathbb{A}_{n, \lambda}^{+}} Z_{\lambda}\left[\hat{\mathcal{A}}_{t,+, \lambda}^{r, \underline{s}_{\sigma}}\right] .
\end{aligned}
$$

If $\sigma \neq \mathrm{Id}$, then there exist $i<j$ such that $\sigma_{i}>\sigma_{j}$. In this case,

$$
\sum_{\underline{s}_{\mathbb{A}_{n, \lambda}^{+}}} Z_{\lambda}\left[\hat{\mathcal{A}}_{t,+, \lambda}^{r, \underline{s}_{\sigma}}\right] \leq \chi_{t, \lambda}^{+}\left(r_{i}, r_{j}\right) \prod_{k \neq i, j} Z_{t,+, \lambda}^{r_{k}, \emptyset}
$$

where $Z_{t,+, \lambda}^{r, \emptyset}$ were defined in (4.2) and, for $\underline{r}, \underline{s} \in \mathbb{A}_{2, \lambda}^{+}$, we define $\underline{s}^{*}=\left(s_{1}, s_{2}\right)^{*}=$ $\left(s_{2}, s_{1}\right)$ and

$$
\chi_{t, \lambda}^{+}(\underline{r})=\sum_{\underline{s} \in \mathbb{A}_{2, \lambda}^{+}} Z_{\lambda}\left[\hat{\mathcal{A}}_{t,+, \lambda}^{\underline{r}, \underline{s}^{*}}\right]
$$

In view of Lemma 4.2, (4.8) would follow once we check that

$$
\lim _{\lambda \downarrow 0} h_{\lambda}^{2} \sum_{\underline{r} \in \mathbb{A}_{2, \lambda}^{+}} \chi_{t, \lambda}^{+}(\underline{r})=0
$$

Given $K>0$, let us define

$$
\epsilon_{\lambda}(K)=\sup _{t} h_{\lambda} \sum_{\substack{r \in \mathbb{N}_{\lambda} \\ r \geq K}} \hat{Z}_{t,+, \lambda}^{r, \emptyset}
$$

By (4.3) of Lemma 4.2,

$$
h_{\lambda}^{2} \sum_{\underline{r} \in \mathbb{A}_{2, \lambda}^{+}} \chi_{t, \lambda}^{+}(\underline{r}) \leq \epsilon_{\lambda}^{2}(K)+2 \epsilon_{\lambda}(K) c_{2}\left(t_{0}\right)+h_{\lambda}^{2} \sum_{\substack{\underline{r} \in \mathbb{A}_{2, \lambda}^{+} \\ 0<r_{1}<r_{2} \leq K}} \chi_{t, \lambda}^{+}(\underline{r}) .
$$

Next, by (4.4) of Lemma 4.2, the term $\epsilon_{\lambda}(K) \rightarrow 0$ as $K$ tends to infinity, uniformly in $\lambda$ small enough. Moreover, choosing $\delta=\frac{t}{K^{2}} \geq \frac{t_{0}}{K^{2}} \triangleq \delta_{0}$, we infer from Lemma 4.3 that

$$
\lim _{\lambda \downarrow 0} \sup _{t \geq t_{0}} \max _{0<r_{1}<r_{2} \leq K} \chi_{t, \lambda}^{+}(\underline{r})=0,
$$

and hence the third term in (4.13) tends to zero (as $\lambda$ tends to 0 ) for any $K$ fixed. (4.11) follows. 
4.3. Proof of Theorem 3.1 (b). Fix $t_{0}>0$. We should check that

$$
\lim _{\lambda \downarrow 0} \sup _{\left\|g_{\lambda}\right\|_{2, \lambda}=\left\|f_{\lambda}\right\|_{2, \lambda}=1} h_{\lambda}^{n} \sum_{\underline{r}, \underline{s} \in \mathbb{A}_{n, \lambda}^{+}} g_{\lambda}(\underline{r}) Z_{\lambda}\left[\hat{\mathcal{A}}_{t,+, \lambda}^{r, \underline{s}} \backslash \hat{\mathcal{P}}_{t,+, \lambda}^{\underline{r}, \underline{s}}\right] f_{\lambda}(\underline{s})=0,
$$

uniformly in $t>t_{0}$. By definition, any path $\underline{x}^{\lambda} \in \hat{\mathcal{A}}_{t,+, \lambda}^{\underline{r}, \underline{s}} \backslash \hat{\mathcal{P}}_{t,+, \lambda}^{\underline{r}, \underline{s}}$ (of the random walk in discrete $\mathbb{Z}_{\lambda}$-time, rescaled as in (3.15)) has to exit $\mathbb{A}_{n, \lambda}^{+}$on its way from $\underline{r}$ to $\underline{s}$. Let $\tau_{-}$and $\tau_{+}, \tau_{ \pm} \in \mathbb{Z}_{\lambda}$, be, respectively, the times of the first and the last visits to $\left\{\mathbb{A}_{n, \lambda}^{+}\right\}^{c}$. Again, by definition of $\hat{\mathcal{A}}_{t,+, \lambda}^{\underline{r}, \underline{s}}$, the points $\underline{x}^{\lambda}\left(\tau_{ \pm}\right)$belong to

$$
\left[\mathbb{A}_{n, \lambda}^{+}\right]_{\sigma_{ \pm}}=\left\{\underline{\mathrm{w}}_{\sigma_{ \pm}}: \underline{\mathrm{w}} \in \mathbb{A}_{n, \lambda}^{+}\right\}
$$

for some permutation $\sigma_{ \pm} \neq \mathrm{Id}$, which of course depends on the particular realization of $\underline{x}^{\lambda}$. Since either

$$
\text { (i) } \tau_{-} \leq t / 2 \text { or } \quad \text { (ii) } t-\tau_{+} \leq t / 2 \text {, }
$$

(4.15) follows by the same arguments as employed for the proof of (3.31) (although, in case (ii), the latter should be applied to the reversed walk).

Indeed, let us fix a permutation $\sigma \neq \mathrm{Id}$. Consider the following modification of (4.7): Set $\eta=\sigma^{-1}$ and

$$
u_{\lambda}(\underline{r})=\max _{u \in[t / 2, t]} \sum_{\underline{s} \in \mathbb{A}_{n, \lambda}^{+}} Z_{\lambda}\left[\hat{\mathcal{A}}_{u,+, \lambda}^{\underline{r}, \underline{\underline{s}} \eta}\right] f_{\lambda}(\underline{s}) .
$$

For $\underline{r}, \underline{s} \in \mathbb{A}_{n, \lambda}^{+}$define,

$$
\rho_{\lambda}(\underline{r}, \underline{s})=Z_{\lambda}\left[\underline{x}^{\lambda}(0)=\underline{r}, \underline{x}^{\lambda}\left(\tau_{-}\right)=\underline{s}_{\sigma}\right] .
$$

Above, $\left\{\underline{x}^{\lambda}(0)=\underline{r}, \underline{x}^{\lambda}\left(\tau_{-}\right)=\underline{s}_{\sigma}\right\}$ is the set of trajectories started at time zero in $\underline{r}$ and arriving, at their first exit from $\mathbb{A}_{n, \lambda}^{+}$, to the point $\underline{s}_{\sigma} \in\left[\mathbb{A}_{n, \lambda}^{+}\right]_{\sigma}$. Clearly, for any $\lambda \geq 0$

$$
\sum_{\underline{s}} \rho_{\lambda}(\underline{r}, \underline{s}) \leq 1
$$

Furthermore, under Assumption (3.14), there exists a constant $c_{3}$ such that

$$
\sum_{\underline{r}} \rho_{\lambda}(\underline{r}, \underline{s}) \leq c_{3}
$$

for all $\lambda$ small enough.

We can now bound from above the contribution of (i) with $x^{\lambda}\left(\tau_{-}\right) \in\left[\mathbb{A}_{n, \lambda}^{+}\right]_{\sigma}$ to the sum in (4.15): applying the Cauchy-Schwarz inequality and the bounds (4.17) and (4.18),

$$
h_{\lambda}^{n} \sum_{\underline{r}, \underline{s}} g_{\lambda}(\underline{r}) \rho_{\lambda}(\underline{r}, \underline{s}) u_{\lambda}(\underline{s}) \leq \sqrt{c_{3}}\left\|g_{\lambda}\right\|_{2, \lambda}\left\|u_{\lambda}\right\|_{2, \lambda}
$$

and one can proceed as in the Proof of Theorem 3.1 (a) to show that $\lim _{\lambda \downarrow 0}\left\|u_{\lambda}\right\|_{2, \lambda}=$ 0 , uniformly in $f$ such that $\left\|f_{\lambda}\right\|_{2, \lambda} \leq 1$. 


\section{Proof of Propositions 3.1 And 3.2}

5.1. Proof of Proposition 3.1. Let us consider $f \in \mathrm{C}_{0}\left(\mathbb{R}_{+}^{n}\right)$ and $f_{\lambda} \in \ell_{2, \lambda}\left(\mathbb{N}_{\lambda}^{n}\right)$. The convergence $\lim _{\lambda \downarrow 0} f_{\lambda}=f$ is still defined via (3.24) with

$$
\rho_{\lambda, n} u(\underline{r})=\frac{1}{h_{\lambda}^{n}} \int_{\left(r_{1}-h_{\lambda}\right)_{+}}^{r_{1}} \ldots \int_{\left(r_{n}-h_{\lambda}\right)_{+}}^{r_{n}} u(\underline{s}) \mathrm{d} \underline{s} .
$$

If $u_{\lambda} \in \ell_{2}\left(\mathbb{N}_{\lambda}^{n}\right)$ converges to $u \in \mathbb{L}_{2}\left(\mathbb{R}_{+}^{n}\right)$, then, evidently, $\tilde{u}_{\lambda} \triangleq u_{\lambda} \mathbb{1}_{\mathbb{A}_{n, \lambda}^{+}} \in \ell_{2}\left(\mathbb{A}_{n, \lambda}^{+}\right)$ converges to $\tilde{u} \triangleq u \mathbb{1}_{\mathbb{A}_{n}^{+}} \in \mathbb{L}_{2}\left(\mathbb{A}_{n}^{+}\right)$. Hence, (3.28) will follow if we check that

$$
\lim _{\lambda \downarrow 0} \sum_{\underline{s} \in \mathbb{N}_{\lambda}^{n}} \prod_{i=1}^{n} \hat{Z}_{t,+, \lambda}^{r_{i}, s_{i}} f_{\lambda}(\underline{s})=\int_{\mathbb{R}_{+}^{n}} \prod_{i=1}^{n} h_{t}\left(r_{i}, s_{i}\right) f(\underline{s}) \mathrm{d} \underline{s},
$$

whenever $f \in \mathrm{C}_{0}\left(\mathbb{R}_{+}^{n}\right)$ and $\lim _{\lambda \downarrow 0} f_{\lambda}=f$.

Next, we may assume without loss of generality that $f_{\lambda}=\rho_{\lambda, n} f$. Hence, there exists $R>0$ such that both $f$ and $f_{\lambda}$ vanish for $r \notin[0, R]^{n} \triangleq \Omega_{R}$. In other words, we can restrict our attention to $f_{\lambda} \in \ell_{2, \lambda}\left(\mathbb{N}_{\lambda}^{n} \cap \Omega_{R}\right)$ and $f \in \mathrm{C}_{0}\left(\Omega_{R}\right)$.

The rest is a monotone class argument based on (3.26): Let $\mathcal{H}_{R}$ be the family of bounded measurable functions on $\Omega_{R}$ such that (5.2) holds. By (3.26), the family $\mathcal{H}_{R}$ contains all the products $\prod_{i=1}^{n} f_{i}\left(r_{i}\right)$ of bounded and measurable functions $f_{1}, \ldots, f_{n}$ on $[0, R]$. In particular, $\mathbb{1}_{\Omega_{R}} \in \mathcal{H}_{R}$. Next, by linearity, $f, g \in \mathcal{H}_{R}$ clearly implies that $a f+b g \in \mathcal{H}_{R}$ for any $a, b \in \mathbb{R}$. Finally, if

$$
0 \leq f^{(1)} \leq f^{(2)} \leq \ldots
$$

is a non-decreasing family of functions from $\mathcal{H}_{R}$ and if $f=\lim f^{(k)}$ exists and is bounded, then $\lim _{k \rightarrow \infty}\left\|f-f^{(k)}\right\|_{2}=0$. Since $\rho_{\lambda, n}$ are contractions, $\left\|f_{\lambda}-f_{\lambda}^{(k)}\right\|_{2, \lambda} \leq$ $\left\|f-f^{(k)}\right\|_{2}$ for all $\lambda>0$. On the one hand, in view of Lemma 4.1 and (4.2), an application of the Cauchy-Schwarz inequality yields

$$
\begin{aligned}
h_{\lambda}^{n} \sum_{\underline{r}}\left(\sum_{\underline{s}} \prod_{i=1}^{n} \hat{Z}_{t,+, \lambda}^{r_{i}, s_{i}} u_{\lambda}(\underline{s})\right)^{2} & \leq\left(h_{\lambda}^{n} \sum_{\underline{r}, \underline{s}} \prod_{i=1}^{n} \hat{Z}_{t,+, \lambda}^{r_{i}, s_{i}}\right)\left(c_{1}\left(t_{0}\right)^{n} h_{\lambda}^{n} \sum_{\underline{s}} u_{\lambda}(\underline{s})^{2}\right) \\
& \leq\left(c_{1}\left(t_{0}\right) c_{2}\left(t_{0}\right)\right)^{n}\left\|u_{\lambda}\right\|_{2, \lambda}^{2},
\end{aligned}
$$

uniformly in $t \geq t_{0}$. In particular,

$$
\left\|\sum_{\underline{s}} \prod_{i=1}^{n} \hat{Z}_{t,+, \lambda}^{r_{i}, s_{i}}\left(f_{\lambda}(\underline{s})-f_{\lambda}^{(k)}(\underline{s})\right)\right\|_{2, \lambda} \leq\left(c_{1}\left(t_{0}\right) c_{2}\left(t_{0}\right)\right)^{n}\left\|f-f^{(k)}\right\|_{2},
$$

uniformly in $t \geq t_{0}$. On the other hand,

$$
\left\|\int_{\mathbb{R}_{+}^{n}} \prod_{i=1}^{n} h_{t}\left(r_{i}, s_{i}\right)\left(f(\underline{s})-f^{(k)}(\underline{s})\right) \mathrm{d} \underline{s}\right\|_{2} \leq\left\|f-f^{(k)}\right\|_{2} .
$$

(5.2) follows, for instance, by a diagonal procedure. 
5.2. Proof of Proposition 3.2. Note that our proof of Theorem 3.3 below, and hence our proof of Proposition 3.3, does not rely on the tightness property which we are trying to establish here. By Proposition 3.3 the one-dimensional projections of $\mathbb{P}_{a_{N}, \underline{r},+, \lambda_{N}}$, that is, the distributions of $\underline{x}^{\lambda_{N}}(t)$ for each fixed $|t| \leq T$, converge.

Then, according to [1, Theorem 8.3], the family $\left\{\mathbb{P}_{a_{N},+, \lambda_{N}}^{\underline{r}, T}\right\}$ is tight if for all positive $\gamma$ and $\beta$ there exist $\delta \in(0,1)$ and $N_{0}$ such that, uniformly in $t \in[-T, T]$,

$$
\mathbb{P}_{a_{N}, \underline{r}, \underline{s} ; T}^{, T}\left(\sup _{s \in[t, t+\delta]}\left|\underline{x}^{\lambda_{N}}(s)-\underline{x}^{\lambda_{N}}(t)\right| \geq \gamma\right) \leq \delta \beta, \quad N \geq N_{0}
$$

Since $a_{N}$ tends to infinity, it suffices to prove (5.4) for $t=0$.

Recall that $T$ is fixed. We may assume that $a_{N} \gg T$. Now, the exponential mixing bound (3.35) implies that the following holds uniformly in $M \geq 2 T$ :

$$
\begin{aligned}
& \mathbb{P}_{a_{N}, \underline{r}, \underline{s},+, \lambda_{N}}\left(\sup _{s \in[0, \delta]}\left|\underline{x}^{\lambda_{N}}(s)-\underline{x}^{\lambda_{N}}(0)\right| \geq \gamma\right) \\
& \leq e^{-c_{1} M}+\mathbb{P}_{M,+, \lambda_{N}}^{g, f ; T}\left(\sup _{s \in[0, \delta]}\left|\underline{x}^{\lambda_{N}}(s)-\underline{x}^{\lambda_{N}}(0)\right| \geq \gamma\right) .
\end{aligned}
$$

Since the potentials $V_{\lambda_{N}}$ in the definition of tilted measures are non-negative, the latter probability is controlled in terms of the underlying random walk:

$$
\mathbb{P}_{M,+, \lambda_{N}}^{g, f ; T}\left(\sup _{s \in[0, \delta]}\left|\underline{x}^{\lambda_{N}}(s)-\underline{x}^{\lambda_{N}}(0)\right| \geq \gamma\right) \leq \frac{1}{Z_{M,+, \lambda_{N}}^{g, f}} \mathbf{P}_{\lambda_{N}}\left(\sup _{s \in[0, \delta]}\left|\underline{x}^{\lambda_{N}}(s)-\underline{x}^{\lambda_{N}}(0)\right| \geq \gamma\right) .
$$

It follows from Theorem 3.2 and the definition of the kernel $\kappa_{t}$ that there exists $c_{2}=c_{2}(g, f)$ such that

$$
Z_{M,+, \lambda_{N}}^{g, f} \geq e^{-c_{2} M}
$$

From these estimates and (5.5), we conclude that

$$
\begin{aligned}
\mathbb{P}_{a_{N},+, \lambda_{N} ; T}^{r}\left(\sup _{s \in[0, \delta]}\left|\underline{x}^{\lambda_{N}}(s)-\underline{x}^{\lambda_{N}}(0)\right|\right. & \geq \gamma) \\
& \leq e^{-c_{1} M}+e^{c_{2} M} \mathbf{P}_{\lambda_{N}}\left(\sup _{s \in[0, \delta]}\left|\underline{x}^{\lambda_{N}}(s)-\underline{x}^{\lambda_{N}}(0)\right| \geq \gamma\right) .
\end{aligned}
$$

An application of the standard functional CLT (recall our assumption (3.3) on unit variance) yields the inequality

$$
\limsup _{N \rightarrow \infty} \mathbf{P}_{\lambda_{N}}\left(\sup _{s \in[0, \delta]}\left|\underline{x}^{\lambda_{N}}(s)-\underline{x}^{\lambda_{N}}(0)\right| \geq \gamma\right) \leq e^{-\gamma^{2} / 4 \delta} .
$$

Consequently,

$$
\limsup _{N \rightarrow \infty} \mathbb{P}_{a_{N}, \underline{r} ; T}^{\underline{s},+, \lambda_{N}}\left(\sup _{s \in[0, \delta]}\left|\underline{x}^{\lambda_{N}}(s)-\underline{x}^{\lambda_{N}}(0)\right| \geq \gamma\right) \leq e^{-c_{1} M}+e^{c_{2} M-\gamma^{2} / 4 \delta}
$$


Choosing $M=\frac{\gamma^{2}}{8 c_{2} \delta}$ (and assuming that the parameters are tuned in such a way that $M \geq 2 T)$, we finally obtain

$$
\limsup _{N \rightarrow \infty} \mathbb{P}_{a_{N}, \underline{r}, T}^{r, T}, \lambda_{N}\left(\sup _{s \in[0, \delta]}\left|\underline{x}^{\lambda_{N}}(s)-\underline{x}^{\lambda_{N}}(0)\right| \geq \gamma\right) \leq 2 e^{-c_{3} \gamma^{2} / 8 \delta},
$$

where $c_{3}=\min \left\{\frac{c_{1}}{c_{2}}, 1\right\}$. Thus, (5.4) is proved.

\section{Proof of Theorem 3.3}

Throughout this section, we shall assume that $H_{\lambda}^{2} \in \mathbb{N}$; this implies that $\mathbb{Z} \subset \mathbb{Z}_{\lambda}$. In particular, the values of rescaled walks $\underline{x}^{\lambda}(\ell)$ in discrete $\mathbb{Z}_{\lambda}$-time are well defined for any $\ell \in \mathbb{Z}$.

6.1. Regular set $\mathbb{A}_{n}^{+, r}$, regular intervals and good blocks. Fix $\eta<\infty$ large enough and $\epsilon>0$ small enough. The regular subset $\mathbb{A}_{n}^{+, r} \subset \mathbb{A}_{n}^{+}$is defined as (under the convention that $\left.x_{0} \equiv 0\right)$ :

$$
\mathbb{A}_{n}^{+, r}=\left\{\underline{x} \in \mathbb{A}_{n}^{+}: x_{n} \leq \eta \quad \text { and } \quad \min _{i \leq n}\left(x_{i}-x_{i-1}\right) \geq \epsilon\right\} .
$$

The notion of regular interval is defined relative to a given continuous $\mathbb{A}_{n}^{+}$-valued function $\underline{x}(\cdot)$. Namely, an interval $[\ell, \ell+1]$ is said to be regular if

$$
\underline{x}(\ell), \underline{x}(\ell+1) \in \mathbb{A}_{n}^{+, r} \text { and } \max _{t \in[\ell, \ell+1]} x_{n}(t) \leq 2 \eta .
$$

Consider now the intervals $D_{\ell}=[2 \ell, 2(\ell+1)]$, which we shall call blocks. A block is a union of two successive unit length intervals,

$$
D_{\ell}=[2 \ell, 2 \ell+1] \cup[2 \ell+1,2(\ell+1)] \triangleq D_{\ell}^{-} \cup D_{\ell}^{+} .
$$

We shall say that $D_{\ell}$ is good if both $D_{\ell}^{+}$and $D_{\ell}^{-}$are regular. If the notion of goodness is defined with respect to random trajectories, namely $\underline{x}^{\lambda}(\cdot)$, then we shall also use $D_{\ell}$ for the corresponding event.

Lemma 6.1. Define $\mathbb{A}_{n, \lambda}^{+, r}=\mathbb{A}_{n}^{+, r} \cap \mathbb{A}_{n, \lambda}^{+}$. There exist two constants $c_{1}, c_{2}$ such that

$$
c_{1} h_{\lambda}^{n} \leq \mathbb{P}_{0,2}^{\underline{r}, \underline{z},+, \lambda}\left(\underline{x}^{\lambda}(1)=\underline{s} \mid D_{0}\right) \leq c_{2} h_{\lambda}^{n},
$$

uniformly in $\lambda$ small and in $\underline{r}, \underline{s}, \underline{z} \in \mathbb{A}_{n, \lambda}^{+, r}$.

We prove Lemma 6.1 in Subsection 7.2.

6.2. Good blocks for a couple of trajectories. Consider now a couple of independent trajectories $\left(\underline{x}^{\lambda}(\cdot), \underline{y}^{\lambda}(\cdot)\right)$ (rescaled as in $(3.15)$ ), distributed according to

$$
\mathbb{P}_{a,+, \lambda}^{\underline{r}, \underline{s}} \otimes \mathbb{P}_{b,+, \lambda}^{\underline{u}, \underline{w}} .
$$

Set $3 M=\min \{a, b\}$. For $D_{\ell} \subset[-2 M, 2 M]$, let us define

$$
\mathfrak{D}_{\ell}=\left\{D_{\ell} \text { is good for both } \underline{x}^{\lambda} \text { and } \underline{y}^{\lambda}\right\} \quad \text { and } \quad \mathcal{M}_{0}=\sum_{-M \leq \ell \leq M-1} \mathbb{1}_{\mathfrak{D}_{\ell}} .
$$




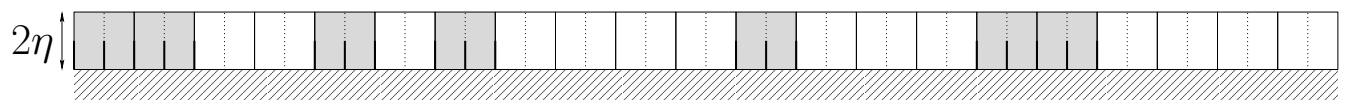

FiguRE 1. Decomposition of the line into blocks. The shaded blocks represent jointly good blocks. Note that, in this case, the couple $\left(\underline{x}^{\lambda}(\cdot), y^{\lambda}(\cdot)\right)$ must be such that all trajectories stay inside the shaded area above jointly good blocks and cross the bold line segments in such a way that their $n$ paths $x_{1}^{\lambda}, \ldots, x_{n}^{\lambda}$, resp. $y_{1}^{\lambda}, \ldots, y_{n}^{\lambda}$, are $\epsilon$-separated. Consequently, $\underline{x}^{\lambda}(\cdot)$ and $\left.\underline{y}^{\lambda}(\cdot)\right)$ can be coupled with positive probability, independently over each such jointly good block.

Lemma 6.2. There exist $\nu>0$ and $\kappa>0$ such that

$$
\mathbb{P}_{a,+, \lambda}^{\underline{r}, \underline{s}} \otimes \mathbb{P}_{b,+, \lambda}^{\underline{u}, \underline{w}}\left(\mathcal{M}_{0} \leq \nu M\right) \leq \mathrm{e}^{-\kappa M},
$$

uniformly in $\lambda$ small, $M$ large and $r_{n}, s_{n}, u_{n}, v_{n} \leq C$.

The proof of Lemma 6.2 is relegated to Subsection 7.3.

6.3. A coupling argument. Fix $\lambda$ small, a negative integer $a<-2 T$ and $\underline{r} \in \mathbb{A}_{n, \lambda}^{+, r}$. For $K \in \mathbb{N}$ and $\underline{u}, \underline{v} \in \mathbb{A}_{n, \lambda}^{+, r}$, define

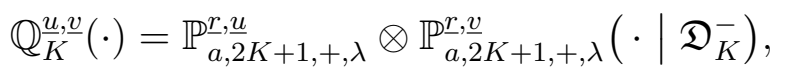

where, similarly to (6.4), we define

$$
\mathfrak{D}_{\ell}^{ \pm}=\left\{D_{\ell}^{ \pm} \text {is regular for both } \underline{x}^{\lambda} \text { and } \underline{y}^{\lambda}\right\} .
$$

In this way, $\mathfrak{D}_{\ell}=\mathfrak{D}_{\ell}^{-} \cap \mathfrak{D}_{\ell}^{+}$. As before, the number $\mathcal{M}_{0}$ of good blocks $D_{\ell}$ for $\ell \in\{1, \ldots, K-1\}$ is defined by

$$
\mathcal{M}_{0}=\sum_{\ell=1}^{K-1} \mathbb{1}_{\mathfrak{D}_{\ell}} .
$$

Let $\mathcal{F}_{T}=\mathcal{F}_{T}^{\lambda}$ be the $\sigma$-algebra generated by rescaled trajectories $(3.15)$ on $[-2 T, 0]$. For the $\sigma$-algebra generated by a couple of such trajectories $\left(\underline{x}^{\lambda}(\cdot), \underline{y}^{\lambda}(\cdot)\right)$, we use $\mathcal{F}_{T} \times \mathcal{F}_{T}$. Given $A \in \mathcal{F}_{T}, A \times \Omega$ stands for the event that $\underline{x}^{\lambda}(\cdot) \in A$ without further restrictions on $y^{\lambda}(\cdot) ; \Omega \times A$ is defined similarly. Define

$$
\psi(m)=\sup _{K>m} \sup _{\underline{u}, \underline{v} \in \mathbb{A}_{n, \lambda}^{+, r}} \sup _{A \in \mathcal{F}_{T}}\left\{\mathbb{Q}_{K}^{\underline{u}, \underline{v}}\left(A \times \Omega ; \mathcal{M}_{0} \geq m\right)-\mathbb{Q}_{K}^{\underline{u}, \underline{v}}\left(\Omega \times A ; \mathcal{M}_{0} \geq m\right)\right\} .
$$

Lemma 6.3. There exists $\delta>0$, which does not depend on $\lambda$, a and $\underline{r}$, such that

$$
\psi(m) \leq(1-\delta)^{m}
$$


Proof. The idea of the proof is sketched in Fig. 1.

Let $K, \underline{u}$ and $\underline{v}$ be as above. Define

$$
\tau=\max \left\{\ell<K: \mathfrak{D}_{\ell} \text { occurs }\right\} .
$$

For any $\mathcal{A} \in \mathcal{F}_{T} \times \mathcal{F}_{T}$, consider the decomposition

$$
\mathbb{Q} \frac{u, \underline{v}}{K}\left(\mathcal{A} ; \mathcal{M}_{0} \geq m\right)=\sum_{\ell=1}^{K-1} \mathbb{Q}_{\frac{u}{K}, \underline{v}}\left(\mathcal{A} ; \mathcal{M}_{0} \geq m ; \tau=\ell\right) .
$$

In its turn, let us decompose each summand in (6.10) as

$$
\mathbb{Q}_{K}^{\underline{u}, \underline{v}}\left(\mathcal{A} ; \mathcal{M}_{0} \geq m ; \tau=\ell\right)=\sum_{\underline{x}, \underline{y} \in \mathbb{A}_{n, \lambda}^{+, r}} \mathbb{Q}_{K}^{\underline{u}, \underline{v}}\left(\mathcal{A} ; \mathcal{M}_{0} \geq m ; \tau=\ell ;\{\underline{x}, \underline{y}\}_{\ell}\right),
$$

where we used the shorthand notation

$$
\{\underline{x}, \underline{y}\}_{\ell}=\left\{\underline{x}^{\lambda}(2 \ell+1)=\underline{x} ; \underline{y}^{\lambda}(2 \ell+1)=\underline{y}\right\} .
$$

Now the Markov property implies that

$$
\mathbb{Q}^{\underline{u}, \underline{v}}\left(\mathcal{A} ; \mathcal{M}_{0} \geq m \mid \tau=\ell ;\{\underline{x}, \underline{y}\}_{\ell}\right)=\mathbb{Q}_{\ell}^{\underline{x}, \underline{y}}\left(\mathcal{A} ; \mathcal{M}_{0} \geq m-1\right) .
$$

Therefore,

$$
\begin{aligned}
\mathbb{Q} \frac{u, \underline{v}}{K}\left(\mathcal{A} ; \mathcal{M}_{0} \geq m\right)=\sum_{\ell=1}^{K-1} \sum_{\underline{x}, \underline{y} \in \mathbb{A}_{n, \lambda}^{+, r}} \mathbb{Q}_{\ell}^{\underline{x}, \underline{y}}\left(\mathcal{A} ; \mathcal{M}_{0} \geq m-1\right) \\
\quad \times \mathbb{Q}^{\underline{u}, \underline{v}}\left(\{\underline{x}, \underline{y}\}_{\ell} \mid \tau=\ell\right) \mathbb{Q}_{\underline{K}}^{\underline{u}, \underline{v}}(\tau=\ell) .
\end{aligned}
$$

This means that, for any $A \in \mathcal{F}_{T}$, and for any $m, K, \underline{u}, \underline{v}$ in question, the following holds:

$$
\begin{array}{r}
\mathbb{Q}_{K}^{\underline{u}, \underline{v}}\left(A \times \Omega ; \mathcal{M}_{0} \geq m\right)-\mathbb{Q}_{\frac{u}{K}, \underline{v}}\left(\Omega \times A ; \mathcal{M}_{0} \geq m\right) \\
=\sum_{\ell, \underline{x}, \underline{y}}\left\{\mathbb{Q}_{\ell}^{\underline{x}, \underline{y}}\left(A \times \Omega ; \mathcal{M}_{0} \geq m-1\right)-\mathbb{Q}_{\ell}^{\underline{x}, \underline{y}}\left(\Omega \times A ; \mathcal{M}_{0} \geq m-1\right)\right\} \\
\quad \times \mathbb{Q}^{\underline{u}, \underline{v}}\left(\{\underline{x}, \underline{y}\}_{\ell} \mid \tau=\ell\right) \mathbb{Q}^{\frac{u}{K}} \underline{v}(\tau=\ell) .
\end{array}
$$

Since, evidently,

$$
\mathbb{Q}_{\ell}^{\underline{x}, \underline{y}}\left(A \times \Omega ; \mathcal{M}_{0} \geq m-1\right)=\mathbb{Q}_{\ell}^{\underline{y}, \underline{x}}\left(\Omega \times A ; \mathcal{M}_{0} \geq m-1\right),
$$

all the terms with $\underline{x}=\underline{y}$ in (6.14) vanish. On the other hand, each unordered pair $\underline{x} \neq \underline{y}$ is encountered exactly twice. Hence, again in view of (6.15), the contribution of each unordered pair $\underline{x} \neq \underline{y}$ to the right-hand side of (6.14) is equal to

$$
\begin{aligned}
\left\{\mathbb { Q } _ { \ell } ^ { \underline { x } , \underline { y } } \left(A \times \Omega ; \mathcal{M}_{0} \geq m\right.\right. & \left.-1)-\mathbb{Q}_{\ell}^{\underline{x}, \underline{y}}\left(\Omega \times A ; \mathcal{M}_{0} \geq m-1\right)\right\} \\
& \times\left\{\mathbb{Q}_{K}^{\underline{u}, \underline{v}}\left(\{\underline{x}, \underline{y}\}_{\ell} \mid \tau=\ell\right)-\mathbb{Q}_{K}^{\underline{u}, \underline{v}}\left(\{\underline{y}, \underline{x}\}_{\ell} \mid \tau=\ell\right)\right\} .
\end{aligned}
$$


On the other hand, by Lemma 6.1,

$$
c_{1} \leq \frac{\mathbb{Q}^{\underline{u}, \underline{v}}\left(\{\underline{x}, \underline{y}\}_{\ell} \mid \tau=\ell\right)}{\mathbb{Q}_{K}^{\underline{u}} \underline{\underline{v}}\left(\{\underline{y}, \underline{x}\}_{\ell} \mid \tau=\ell\right)} \leq c_{2},
$$

uniformly in all the situations in question. Set $\delta=\frac{c_{1}}{c_{2}}$. Then, (6.17) implies that the expression in (6.16) is bounded above by

$$
\begin{aligned}
& \psi(m-1)(1-\delta) \max \left\{\mathbb{Q}_{\frac{u}{K}}^{\underline{v}}\left(\{\underline{x}, \underline{y}\}_{\ell} \mid \tau=\ell\right), \mathbb{Q}^{\frac{u}{K}, \underline{v}}\left(\{\underline{y}, \underline{x}\}_{\ell} \mid \tau=\ell\right)\right\} \\
& \leq \psi(m-1)(1-\delta)\left\{\mathbb{Q} \frac{\underline{u}, \underline{v}}{K}\left(\{\underline{x}, \underline{y}\}_{\ell} \mid \tau=\ell\right)+\mathbb{Q}^{\underline{u}, \underline{v}}\left(\{\underline{y}, \underline{x}\}_{\ell} \mid \tau=\ell\right)\right\} .
\end{aligned}
$$

Since $\mathbb{Q}_{K}^{\underline{u}, \underline{v}}$ is a probability measure, substituting (6.18) into (6.14) yields the conclusion (6.9) of the lemma.

6.4. Conclusion of the proof. We are in a position to conclude the proof of Theorem 3.3. Let $a, b \geq(T+K)$ and $\underline{r}, \underline{s}, \underline{w}, \underline{z} \in \mathbb{A}_{n, \lambda}^{+}$with $r_{n}, s_{n}, w_{n}, z_{n} \leq C$. Let $A$ be an event generated by the rescaled trajectories of $(3.15)$ on $[-T, T]$. Then,

$$
\mathbb{P}_{a,+, \lambda}^{\underline{r}, \underline{s}}(A)-\mathbb{P}_{b,+, \lambda}^{\underline{w}, \underline{z}}(A)=\mathbb{P}_{a,+, \underline{r}^{\prime}, \lambda}^{\underline{s}} \otimes \mathbb{P}_{b,+, \lambda}^{\underline{w}, \underline{z}}\left(\mathbb{1}_{A \times \Omega}-\mathbb{1}_{\Omega \times A}\right) .
$$

Consider a pair of trajectories $\left(x^{\lambda}(\cdot), y^{\lambda}(\cdot)\right)$, sampled from $\mathbb{P}_{a,+,+, \lambda}^{\underline{r}, \underline{s}} \otimes \mathbb{P}_{b,+, \lambda}^{\underline{w}, \underline{z}}$. Let $\mathcal{M}_{ \pm}$ be the number of jointly good blocks $D_{\ell}$ with $T \leq 2 \ell \leq T+K-2$, respectively $-T-K \leq 2 \ell \leq-T-2$.

By Lemma 6.2 , there exist $\nu^{\prime}, \kappa^{\prime}>0$ such that, up to the $2 \mathrm{e}^{-\kappa^{\prime} K}$ correction, we may restrict our attention to the event

$$
E_{K}=\left\{\mathcal{M}_{+} \geq \nu^{\prime} K\right\} \cap\left\{\mathcal{M}_{-} \geq \nu^{\prime} K\right\}
$$

On the other hand, by Lemma 6.3,

$$
\left|\mathbb{P}_{a,+, \lambda}^{\underline{r}, \underline{s}} \otimes \mathbb{P}_{b,+, \lambda}^{\underline{w}, \underline{z}}\left(\mathbb{1}_{E_{K}}\left(\mathbb{1}_{A \times \Omega}-\mathbb{1}_{\Omega \times A}\right)\right)\right| \leq(1-\delta)^{\nu^{\prime} K}
$$

Our target exponential mixing bound (3.35) follows.

\section{Proof of Lemmas 6.1 AND 6.2}

7.1. Probabilistic estimates. Our proofs of Lemma 6.1 and 6.2 rely on strong approximation techniques and on refined information on random walks in Weyl chambers. There are three inputs, (I.1)-(I.3), which are stated below, but proved in the Appendix. In the sequel, we fix $\eta$ sufficiently large; in particular, $\eta>C$, where $C$ is the constant which appears in Theorem A. Furthermore, we fix $\epsilon>0$ sufficiently small.

First of all, we claim that, for any $0<a<b<\infty$, there exists $\nu=\nu(a, b)>0$ such that

$$
\hat{\mathbf{P}}_{t,+, \lambda}^{r}\left(\underline{x}^{\lambda}(t)=\underline{z}, \max _{s \in[0, t]} x_{n}^{\lambda}(s) \leq 2 \eta\right) \geq \nu h_{\lambda}^{n},
$$

uniformly in $t \in[a, b], \underline{r}, \underline{z} \in \mathbb{A}_{n, \lambda}^{+, r}$ and $\lambda$ small. 
Next, let

$$
\tau=\inf \left\{t \geq 0: \underline{x}^{\lambda} \notin \mathbb{A}_{n, \lambda}^{+}\right\}
$$

be the first exit time of the path from $\mathbb{A}_{n, \lambda}^{+}$. We then claim that, for any $0<a<b<$ $\infty$, there exists $\rho=\rho(a, b)$ such that the following two lower bounds hold uniformly in $t \in[a, b], \underline{u} \in \mathbb{A}_{n, \lambda}^{+}$with $u_{n} \leq \eta$ and in $\lambda$ sufficiently small:

$$
\begin{gathered}
\hat{\mathbf{P}}_{t, \lambda}^{u}\left(\max _{s \in[0, t]} x_{n}^{\lambda}(s) \leq 2 \eta \mid \tau>t\right) \geq \rho, \\
\hat{\mathbf{P}}_{t,+, \lambda}^{u}\left(\underline{x}^{\lambda}(t) \in \mathbb{A}_{n, \lambda}^{+, r} \mid \max _{s \in[0, t]} x_{n}^{\lambda}(s) \leq 2 \eta, \tau>t\right) \geq \rho .
\end{gathered}
$$

7.2. Proof of Lemma 6.1. Since, by definition, the area-tilt of every path in $D_{0}$ is uniformly bounded, it suffices to prove the lemma for random walks without area tilts. That is, we need to show that

$$
c_{1} h_{\lambda}^{n} \leq \hat{\mathbf{P}}_{2,+, \lambda}^{\underline{r}, \underline{s}}\left(\underline{x}^{\lambda}(1)=\underline{z} \mid D_{0}\right) \leq c_{2} h_{\lambda}^{n},
$$

uniformly in $\underline{r}, \underline{s}, \underline{z} \in \mathbb{A}_{n, \lambda}^{+, r}$.

We start by noting that upper bounds for $\hat{\mathbf{P}}_{+, \lambda}^{\underline{r}}\left(D_{0}, \underline{x}^{\lambda}(2)=\underline{s}\right)$ and $\hat{\mathbf{P}}_{+, \lambda}^{r}\left(\underline{x}^{\lambda}(1)=\right.$ $\underline{z}, D_{0}, \underline{x}^{\lambda}(2)=\underline{s}$ ) follow from the classical inequalities for concentration functions. Indeed, by [9, Theorem 6.2], there exists a constant $c_{3}$ such that

$$
\hat{\mathbf{P}}_{+, \lambda}^{r}\left(\underline{x}^{\lambda}(t)=\underline{y}\right) \leq \hat{\mathbf{P}}_{\lambda}^{\frac{r}{\lambda}}\left(\underline{x}^{\lambda}(t)=\underline{y}\right) \leq \frac{c_{3}}{t^{n / 2}} h_{\lambda}^{n},
$$

uniformly in $\underline{r}, y$ and $t \geq 0$.

Consequently,

$$
\hat{\mathbf{P}}_{+, \lambda}^{\underline{r}}\left(D_{0}, \underline{x}^{\lambda}(2)=\underline{s}\right) \leq \hat{\mathbf{P}}_{\lambda}^{\underline{r}}\left(\underline{x}^{\lambda}(2)=\underline{s}\right) \leq \frac{c_{3}}{2^{n / 2}} h_{\lambda}^{n}
$$

and

$$
\begin{aligned}
\hat{\mathbf{P}}_{+, \lambda}^{r}\left(\underline{x}^{\lambda}(1)=\underline{z}, D_{0}, \underline{x}^{\lambda}(2)=\underline{s}\right) & \leq \hat{\mathbf{P}}_{\lambda}^{r}\left(\underline{x}^{\lambda}(1)=\underline{z}, \underline{x}^{\lambda}(2)=\underline{s}\right) \\
& =\hat{\mathbf{P}}_{\lambda}^{r}\left(\underline{x}^{\lambda}(1)=\underline{z}\right) \hat{\mathbf{P}}_{\lambda}^{\underline{z}}\left(\underline{x}^{\lambda}(1)=\underline{s}\right) \\
& \leq c_{3}^{2} h_{\lambda}^{2 n},
\end{aligned}
$$

uniformly in $\underline{r}, \underline{s}, \underline{z} \in \mathbb{A}_{n, \lambda}^{+, r}$.

The corresponding matching lower bounds follow from (I.1). Indeed,

$$
\begin{aligned}
\hat{\mathbf{P}}_{+, \lambda}^{\underline{r}}\left(\underline{x}^{\lambda}(1)\right. & \left.=\underline{z}, D_{0}, \underline{x}^{\lambda}(2)=\underline{s}\right) \\
& =\hat{\mathbf{P}}_{+, \lambda}^{\underline{r}}\left(\underline{x}^{\lambda}(1)=\underline{z}, \max _{t \leq 1} x_{n}^{\lambda}(t) \leq 2 \eta\right) \hat{\mathbf{P}}_{+, \lambda}^{\underline{z}}\left(\underline{x}^{\lambda}(1)=\underline{s}, \max _{t \leq 1} x_{n}^{\lambda}(t) \leq 2 \eta\right) \\
& \stackrel{(\text { I.1) }}{\geq} c_{4} h_{\lambda}^{2 n},
\end{aligned}
$$

for any $\underline{r}, \underline{z}, \underline{s} \in \mathbb{A}_{n, \lambda}^{+, r}$. Since the cardinality

$$
\left|\mathbb{A}_{n, \lambda}^{+, r}\right| \geq c_{5}(\epsilon) \eta^{n} h_{\lambda}^{-n}
$$


we infer, by summing over $\underline{z}$ in $(7.6)$, that

$$
\hat{\mathbf{P}}_{+, \lambda}^{r}\left(\underline{x}^{\lambda}(2)=\underline{s}, D_{0}\right) \geq c_{6} h_{\lambda}^{n} .
$$

It remains to note that the lower bound in (7.2) follows from (7.4) and (7.6), and that the upper bound in (7.2) follows from (7.5) and (7.8).

7.3. Proof of Lemma 6.2. The proof of Lemma 6.2 proceeds in two steps.

Consider the 5-blocks

$$
D_{\ell}^{(5)} \triangleq D_{5 \ell-2} \cup D_{5 \ell-1} \cup D_{5 \ell} \cup D_{5 \ell+1} \cup D_{5 \ell+2},
$$

where $\ell \in\{-\lfloor M / 5\rfloor, \ldots,\lfloor M / 5\rfloor\} \subset \mathbb{Z}$.

Let us say that a 5 -block $D_{\ell}^{(5)}$ is pre-good (relative to a trajectory $\left.\underline{x}^{\lambda}(\cdot)\right)$ if both

$$
\min _{t \in D_{5 \ell-2}} x_{n}^{\lambda}(t), \min _{t \in D_{5 \ell+2}} x_{n}^{\lambda}(t) \leq \eta
$$

Given a couple of trajectories $\underline{x}^{\lambda}$ and $\underline{y}^{\lambda}$, let $\tilde{\mathfrak{D}}_{\ell}^{(5)}$ denote the event that $D_{\ell}^{(5)}$ is pre-good for both $\underline{x}^{\lambda}$ and $\underline{y}^{\lambda}$.

STEP 1. Note that the definitions are set up in such a way that $D_{5 \ell}$ is the middle section of $D_{\ell}^{(5)}$. We claim that there exists $\rho_{1}=\rho_{1}(\eta, \epsilon)>0$ such that

$$
\mathbb{P}_{-4,6,+, \lambda}^{r, s}\left(D_{0} \text { is good } \mid D_{0}^{(5)} \text { is pre-good }\right) \geq \rho_{1},
$$

uniformly in $\underline{r}, \underline{s} \in \mathbb{A}_{n, \lambda}^{+}$and $\lambda$ sufficiently small.

As a result, for any $\ell \in\{-\lfloor M / 5\rfloor, \ldots,\lfloor M / 5\rfloor\} \subset \mathbb{Z}$,

$$
\mathbb{P}_{a,+, \lambda}^{\underline{r}, \underline{s}} \otimes \mathbb{P}_{b, \underline{u}, \underline{w}}^{,}\left(\mathfrak{D}_{5 \ell} \mid \tilde{\mathfrak{D}}_{\ell}^{(5)}\right) \geq \rho_{1}^{2},
$$

uniformly in $\underline{r}, \underline{s}, \underline{u}, \underline{v} \in \mathbb{A}_{n, \lambda}^{+}$and $\lambda$ small enough.

By the Markov property, this means that any jointly pre-good 5-block gives rise to a good block in its middle section with probability at least $\rho_{1}^{2}$, regardless of the behavior of trajectories outside this particular pre-good 5-block.

STEP 2. In this second step, we control the density of jointly pre-good 5-blocks $D_{\ell}^{(5)}$ which lie inside $[-2 M, 2 M]$. Define

$$
\mathcal{M}_{0}^{(5)}=\sum_{j=-\lfloor M / 5\rfloor}^{\lfloor M / 5\rfloor} \mathbb{1}_{\mathfrak{D}_{\ell}^{(5)}} .
$$

We claim that there exist $\nu^{(5)}>0$ and $\kappa^{(5)}>0$ such that

$$
\mathbb{P}_{a,+, \lambda}^{\underline{r}, \underline{s}} \otimes \mathbb{P}_{b,+, \lambda}^{\underline{u}, \underline{w}}\left(\mathcal{M}_{0}^{(5)} \leq \nu^{(5)} M\right) \leq \mathrm{e}^{-\kappa^{(5)} M} .
$$

uniformly in $\lambda$ small, $M$ large, $a, b \geq 3 M$ and $r_{n}, s_{n}, u_{n}, v_{n} \leq C$.

Evidently, (7.11) and (7.12) imply the target bound (6.5). 


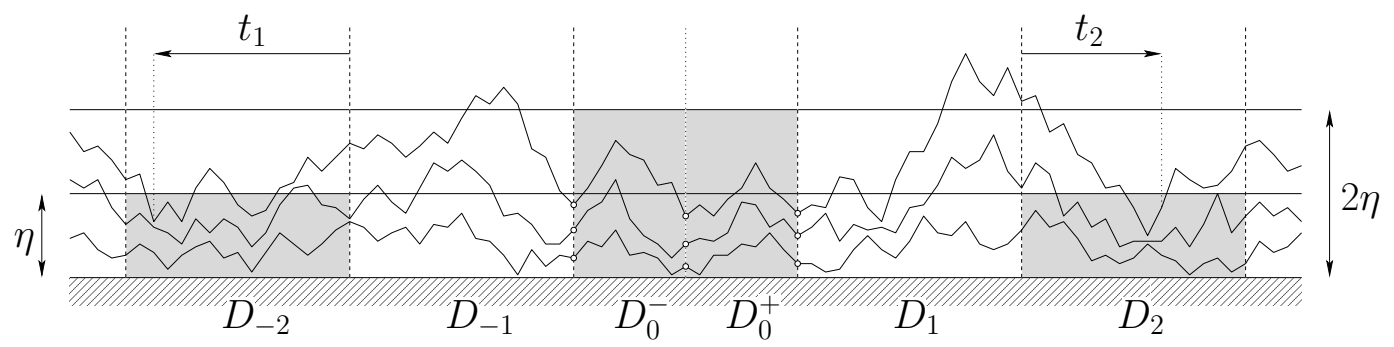

Figure 2. A picture of the 5-block $D_{0}^{(5)}$. In the picture, this 5-block is pre-good, since the top-most path (here $n=3$ ) visits the shaded areas in the blocks $D_{-2}$ and $D_{2}$; the corresponding random variables $t_{1}$ and $t_{2}$ are also represented. The event $D_{0}$ also occurs: the top-most path stays inside the shaded area above the block $D_{0}=D_{0}^{-} \cup D_{0}^{+}$and the $n=3$ paths stay $\epsilon$ apart from each other and the bottom wall at the boundary of $D_{0}^{-}$and $D_{0}^{+}$(the corresponding positions of the 3 paths there are marked with dots).

7.4. Proof of (7.10). We are going to show that

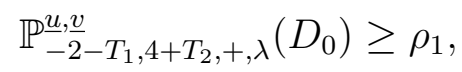

uniformly in $\underline{u}, \underline{v}$ with $u_{n}, v_{n} \leq \eta, T_{1}, T_{2} \in[0,2]$ and $\lambda$ sufficiently small. The target (7.10) is an immediate consequence by the Gibbs property and conditioning on the left-most $t_{-} \in[-4,-2]$ and the right-most $t_{+} \in[4,6]$ such that $x_{n}^{\lambda}\left(t_{-}\right), x_{n}^{\lambda}\left(t_{+}\right) \leq$ $\eta$; see Fig. 7.4.

The proof boils down to deriving an appropriate upper bound on the partition function $Z_{-2}^{\underline{u}, \underline{v}} T_{1}, 4+T_{2},+, \lambda$ and an appropriate matching lower bound on the constrained partition function $Z_{-2}^{\underline{u}, \underline{u}}-T_{1}, 4+T_{2},+, \lambda\left[D_{0}\right]$.

In the sequel, $\underline{\tilde{x}}^{\lambda}$ stands for the reversed random walk with transition probabilities $\tilde{p}_{z}=p_{-z}$. Let $\tilde{\tau}$ be the first exit time of $\underline{\tilde{x}}^{\lambda}$ from $\mathbb{A}_{n, \lambda}^{+, r}$. We assume that the constants $\nu$ and $\rho$ in the probabilistic estimates (I.1)-(I.3) are chosen in such a way that the corresponding bounds hold for the reflected process as well.

STEP 1. (An upper bound on $Z_{-2-T_{1}, 4+T_{2},+, \lambda}^{\underline{u}, \underline{v}}$ ) Since we are dealing with nonnegative potentials,

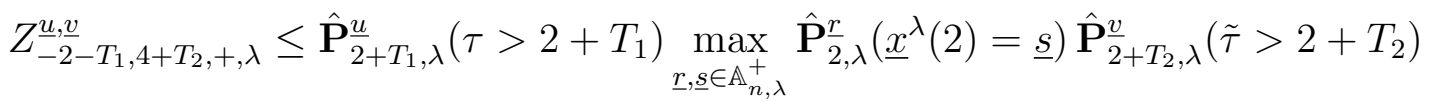

$$
\begin{aligned}
& \leq c_{1} h_{\lambda}^{n} \hat{\mathbf{P}}_{2+T_{1}, \lambda}^{u}\left(\tau>2+T_{1}\right) \hat{\mathbf{P}}_{2+T_{2}, \lambda}^{\underline{v}}\left(\tilde{\tau}>2+T_{2}\right)
\end{aligned}
$$

The second inequality follows from the concentration bound (7.3). 


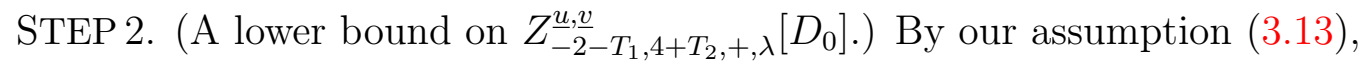

$$
\begin{aligned}
& Z_{-2-T_{1}, 4+T_{2},+, \lambda}^{\underline{u}, \underline{v}}\left(D_{0}\right) \geq \\
& \quad \mathrm{e}^{-10 n q_{0}(2 \eta)} \mathbf{P}_{-2-T_{1}, 4+T_{2},+, \lambda}^{\underline{u}}\left(D_{0} \max _{t \in\left[-2-T_{1}, 4+T_{2}\right]} x_{n}^{\lambda}(t) \leq 2 \eta, \underline{x}^{\lambda}\left(4+T_{2}\right)=\underline{v}\right) .
\end{aligned}
$$

Above $\hat{\mathbf{P}}_{s, t,+, \lambda}^{v}$ is the provisional notation for the restriction of the law of the rescaled walk started at time $s$ at $\underline{v}$ to the set of trajectories which stay inside $\mathbb{A}_{n, \lambda}^{+}$during the time interval $[s, t]$.

The probability on the right-hand side of (7.15) is bounded below by the following product of three factors:

$$
\begin{aligned}
\hat{\mathbf{P}}_{2+T_{1}, \lambda}^{\underline{u}} & \left(\tau>2+T_{1}, \max _{t \leq 2+T_{1}} x_{n}^{\lambda}(t) \leq 2 \eta, \underline{x}^{\lambda}\left(2+T_{1}\right) \in \mathbb{A}_{n, \lambda}^{+, r}\right) \\
& \times \min _{\underline{r}, \underline{s} \in \mathbb{A}_{n, \lambda}^{+, r}} \hat{\mathbf{P}}_{2,+, \lambda}^{\underline{r}}\left(D_{0}, \underline{x}^{\lambda}(2)=\underline{s}\right) \\
& \times \hat{\mathbf{P}}_{2+T_{2}, \lambda}^{v}\left(\tilde{\tau}>2+T_{2}, \max _{t \leq 2+T_{2}} \tilde{x}_{n}^{\lambda}(t) \leq 2 \eta, \underline{\tilde{x}}^{\lambda}\left(2+T_{2}\right) \in \mathbb{A}_{n, \lambda}^{+, r}\right) .
\end{aligned}
$$

On the one hand, in view of (7.8), the middle factor is bounded below by $c_{2} h_{\lambda}^{n}$. On the other hand, the probabilistic bounds (I.2), (I.3) imply that the left-most factor

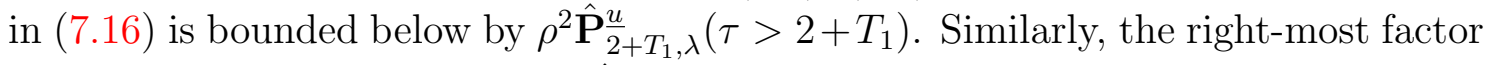
in $(7.16)$ is bounded below by $\rho^{2} \hat{\mathbf{P}}^{\frac{v}{2}}{ }_{T_{2}, \lambda}\left(\tilde{\tau}>2+T_{2}\right)$. Hence,

$$
Z_{-2-T_{1}, 4+T_{2},+, \lambda}^{\underline{u}, \underline{v}}\left[D_{0}\right] \geq c_{3} \rho^{4} h_{\lambda}^{n} \hat{\mathbf{P}}_{2+T_{1}, \lambda}^{\underline{u}}\left(\tau>2+T_{1}\right) \hat{\mathbf{P}}_{2+T_{2}, \lambda}^{\underline{v}}\left(\tilde{\tau}>2+T_{2}\right) .
$$

Since

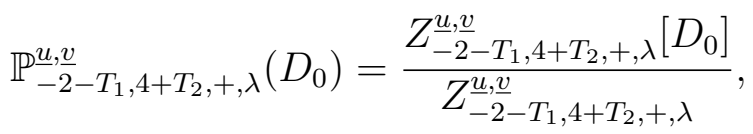

(7.13) directly follows from (7.14) and (7.17).

7.5. Proof of (7.12). We start by deriving a lower bound on partition functions, as this will allow us to exclude sets of pathological trajectories.

Lemma 7.1. There exist constants $c_{1}=c_{1}(n)$ and $c_{2}=c_{2}(n, \eta)$ and a sufficiently large value $T_{0}=T_{0}(\eta)$ such that, for all $T \geq T_{0}$,

$$
Z_{T,+, \lambda}^{\underline{w}, \underline{z}} \geq c_{2} e^{-c_{1} T} \hat{\mathbf{P}}_{2 T,+, \lambda}^{\underline{w}}\left(\underline{x}^{\lambda}(2 T)=\underline{z}\right),
$$

uniformly in $\lambda$ small and in $z_{n}, w_{n} \leq \eta$.

Proof. The point is that constant $c_{1}$ does not depend on $\eta$, only on the dimension $n$. The dependence of $c_{1}$ on $n$ is expressed in terms of the dependence of $\epsilon$ (in the definition of the regular set $\mathbb{A}_{n, \lambda}^{+, r}$, see (6.1)) on $n$. We shall work with a fixed small value of $\epsilon>0$ which satisfies

$$
n \epsilon<1 \text {. }
$$


In the sequel, we consider $T>2$. Let $\mathbb{A}_{n, \lambda}^{+, r}(\alpha)=\mathbb{A}_{n, \lambda}^{+, r} \cap\left\{\underline{x}: x_{n} \leq \alpha\right\}$. Consider the events

$$
\begin{aligned}
& \mathcal{E}_{-}=\left\{\max _{t \in[0,1]} x_{n}^{\lambda}(t) \leq 2 \eta, \underline{x}(1) \in \mathbb{A}_{n, \lambda}^{+, r}(1)\right\}, \\
& \mathcal{E}_{+}=\left\{\max _{t \in[2 T-1,2 T]} x_{n}^{\lambda}(t) \leq 2 \eta, \underline{x}(2 T-1) \in \mathbb{A}_{n, \lambda}^{+, r}(1)\right\}
\end{aligned}
$$

and

$$
\mathcal{E}_{T}=\left\{\max _{t \in[1,2 T-1]} x_{n}^{\lambda}(t)<2\right\} .
$$

On the one hand, by (3.13),

$$
Z_{T,+, \lambda}^{\underline{w}, \underline{z}} \geq \mathrm{e}^{-2 n q_{0}(2 \eta)-2 n q_{0}(2) T} \hat{\mathbf{P}}_{2 T,+, \lambda}^{\underline{w}}\left(\mathcal{E}_{-}, \mathcal{E}_{T}, \mathcal{E}_{+}, \underline{x}^{\lambda}(2 T)=\underline{z}\right) .
$$

On the other hand,

$$
\hat{\mathbf{P}}_{2 T,+, \lambda}^{\underline{w}}\left(\underline{x}^{\lambda}(2 T)=\underline{z}\right) \leq c_{3} \hat{\mathbf{P}}_{1, \lambda}^{\underline{w}}(\tau>1) \hat{\mathbf{P}}_{1, \lambda}^{\underline{z}}(\tilde{\tau}>1) \frac{h_{\lambda}^{n}}{T^{n / 2}} .
$$

Above, we relied on the concentration bound (7.3).

In order to compare the probabilities appearing in (7.21) and (7.22), note that an application of (I.1)-(I.3) (and the observation that, as in (7.7), the cardinality $\left.\left|\mathbb{A}_{n, \lambda}^{+, r}(1)\right| \geq c_{4}(\epsilon) h_{\lambda}^{-n}\right)$ yields

$$
\begin{aligned}
\hat{\mathbf{P}}_{2 T,+, \lambda}^{\underline{w}}\left(\mathcal{E}_{-},\right. & \left.\mathcal{E}_{T}, \mathcal{E}_{+}, \underline{x}^{\lambda}(2 T)=\underline{z}\right) \geq c_{5} \hat{\mathbf{P}}_{1, \lambda}^{w}(\tau>1) \hat{\mathbf{P}}_{1, \lambda}^{\underline{z}}(\tilde{\tau}>1) \\
& \times \min _{\underline{u}, \underline{v} \in \mathbb{A}_{n, \lambda}^{+, r}(1)} \hat{\mathbf{P}}_{2(T-1),+, \lambda}^{u}\left(\max _{t \in[0,2(T-1)]} x_{n}^{\lambda}(t)<2, \underline{x}^{\lambda}(2(T-1))=\underline{v}\right) .
\end{aligned}
$$

However,

$$
\min _{\underline{u}, \underline{v} \in \mathbb{A}_{n, \lambda}^{+, r}(1)} \hat{\mathbf{P}}_{2(T-1),+, \lambda}^{\underline{u}}\left(\max _{t \in[0,2(T-1)]} x_{n}^{\lambda}(t)<2, \underline{x}^{\lambda}(2(T-1))=\underline{v}\right) \geq \mathrm{e}^{-c_{6}(\epsilon) T} h_{\lambda}^{n} .
$$

Indeed, consider $n$ walks $x_{\ell}^{\lambda}, \ell=1, \ldots, n$, which go from $u_{\ell}$ to $v_{\ell}$ inside space-time tubes of width $\epsilon / 4$ centered around the space-time segments $\left[\left(u_{\ell}, 0\right),\left(v_{\ell}, 2(T-1)\right]\right.$. By construction, these walks stay in $\mathbb{A}_{n, \lambda}^{+} \cap\left\{\underline{x}: x_{n}<2\right\}$. By a coarse splitting into time-blocks of lengths of order $\epsilon^{2}$, we bound from below the probability of staying within such tubes by $\mathrm{e}^{-c_{6}(\epsilon) T}$. Applying the local CLT for the last step, we bound from below the probability of ending up in $\underline{z}$ by a multiple of $h_{\lambda}^{n}$. (7.24) follows. The bound (7.18) is a direct consequence of (7.22) and (7.21), (7.23) and (7.24).

Let us resume the proof of (7.12). Without loss of generality, we shall assume that $a=3 M$ and $b \geq a$. In the sequel, the trajectory $\underline{x}^{\lambda}$ is sampled from $\mathbb{P}_{a,+, \lambda}^{\underline{r}, \underline{s}}$ and $\underline{y}^{\lambda}$ is sampled from $\mathbb{P} \frac{u}{b,},+, \lambda$. Recall that $r_{n}, s_{n}, u_{n}, v_{n} \leq C \leq \eta$. 


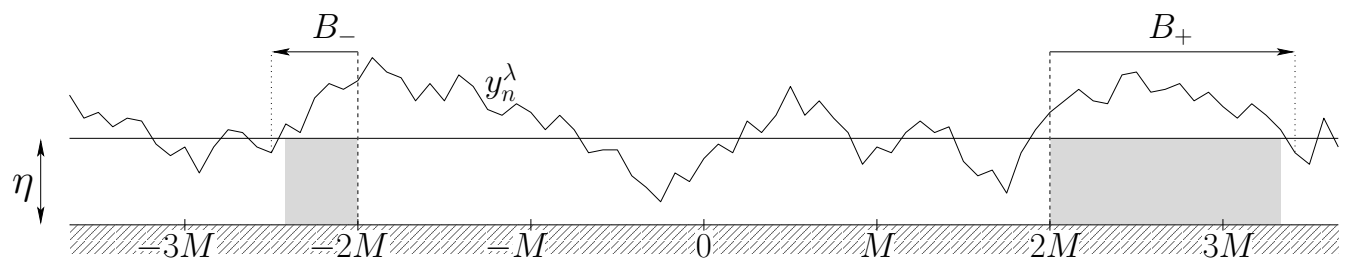

FiguRE 3. The definition of the random variables $B_{-}$and $B_{+}$. Note that the shaded areas are necessarily below the path $y_{n}^{\lambda}$ and thus contribute to the area-tilt.

In principle, $b$ can be much larger than $M$. Let us verify that one can restrict our attention to the case where $b$ is of the same order as $M$. Define the random variables $B_{ \pm} \geq 0$ via (see Figure 7.5 )

$$
-2 M-B_{-}=\max \left\{t \leq-2 M: y_{n}^{\lambda}(t) \leq \eta\right\}
$$

and, accordingly, $2 M+B_{+}=\min \left\{t \geq 2 M: y_{n}^{\lambda}(t) \leq \eta\right\}$. By the Gibbs property,

$$
\begin{aligned}
& \mathbb{P} \frac{u, \underline{v}}{b,+, \lambda}\left(B_{ \pm}=b_{ \pm}\right) \\
& \leq \max _{w_{n}, z_{n} \leq \eta} \mathbb{P}_{-2}^{\underline{w}, \underline{z}}{ }_{-2-b_{-}, 2 M+b_{+},+, \lambda}\left(\min _{t \in\left(-2 M-b_{-},-2 M\right]} x_{n}^{\lambda}(t) \wedge_{t \in\left[2 M, 2 M+b_{+}\right)} x_{n}^{\lambda}(t)>\eta\right) \text {. }
\end{aligned}
$$

Therefore, in view of (3.13),

$$
\mathbb{P} \frac{\underline{u}, \underline{v}}{b,+, \lambda}\left(B_{ \pm}=b_{ \pm}\right) \leq \mathrm{e}^{-\left(b_{-}+b_{+}\right) q_{0}(\eta)} \max _{w_{n}, z_{n} \leq \eta} \frac{\hat{\mathbf{P}}_{T,+, \lambda}^{\underline{w}}\left(\underline{x}^{\lambda}(T)=\underline{z}\right)}{\hat{Z}_{T,+, \lambda}^{w}, \underline{z}}
$$

where we have set $T=4 M+b_{-}+b_{+}$. Using the lower bound (7.18) on $\hat{Z}_{T, \underline{w}, \lambda}^{\underline{w}, \underline{z}}$, we conclude that

$$
\mathbb{P}_{b,+, \lambda}^{\underline{u}, \underline{v}}\left(B_{ \pm}=b_{ \pm}\right) \leq c_{7}(\epsilon) \mathrm{e}^{c_{8}(\epsilon) M-\left(b_{-}+b_{+}\right) q_{0}(\eta)} .
$$

Therefore, if we choose $\eta$ so large that

$$
q_{0}(\eta)>2 c_{8}(\epsilon)
$$

then we may ignore the case $b_{ \pm} \geq M$.

Consequently, (7.12) will follow once we check that

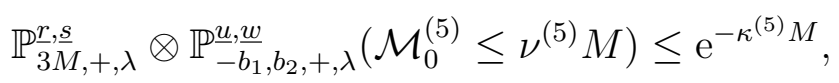

uniformly in $\lambda$ small, $M$ large, $b_{1}, b_{2} \in[2 M, 3 M]$ and $r_{n}, s_{n}, u_{n}, v_{n} \leq \eta$. 
If we choose $\nu^{(5)}$ to be sufficiently small, for instance smaller than 0.2 , then, by $(3.13)$,

$$
\begin{aligned}
\mathbb{P}_{3 M,+, \lambda}^{\underline{r}, \underline{s}} \otimes \mathbb{P}_{b,+, \lambda}^{\underline{u}, \underline{w}}\left(\mathcal{M}_{0}^{(5)}\right. & \left.\leq \nu^{(5)} M\right) \\
\leq & \mathrm{e}^{-\frac{q_{0}(\eta)}{10} M} \frac{\hat{\mathbf{P}}_{6 M,+, \lambda}^{\frac{r}{r}}\left(\underline{x}^{\lambda}(3 M)=\underline{s}\right) \hat{\mathbf{P}}_{b_{1}+b_{2},+, \lambda}^{\underline{u}}\left(\underline{y}^{\lambda}(b)=\underline{w}\right)}{Z_{3}^{\underline{r}, \underline{s}}, Z_{,+, \lambda} Z_{-b_{1}, b_{2},+, \lambda}^{\underline{w}}} .
\end{aligned}
$$

Taking $\eta$ and $M$ large enough and applying (7.18), we arrive to (7.30).

\section{Appendix A. Strong approximation techniques.}

In order to prove (I.1), we are going to apply strong approximation techniques from [6]. By rescaling, it is sufficient to consider the case $t=1$.

In the sequel, $\hat{\mathbf{P}}_{+}^{r}$ denotes the restriction of the law of the $n$-dimensional Brownian motion $\underline{B}$ started at $\underline{r}$ to the set $\mathbb{A}_{n}^{+}$.

Define

$$
O_{\epsilon}(\underline{z})=\left\{\underline{y}:\left|y_{i}-z_{i}\right| \leq \frac{\epsilon}{3} \text { for all } i\right\} .
$$

It follows easily from [6, Lemma 17] that

$$
\begin{aligned}
\hat{\mathbf{P}}_{+, \lambda}^{r}\left(\underline{x}^{\lambda}(1-\gamma) \in O_{\epsilon}(\underline{z}), \max _{t \leq 1-\gamma}\right. & \left.x_{n}^{\lambda}(t) \leq 2 \eta\right) \\
& =\hat{\mathbf{P}}_{+}^{\underline{r}}\left(\underline{B}(1-\gamma) \in O_{\epsilon}(\underline{z}), \max _{t \leq 1-\gamma} B_{n}(t) \leq 2 \eta\right)+o(1),
\end{aligned}
$$

uniformly in $\underline{r}, \underline{z} \in \mathbb{A}_{n, \lambda}^{+, r}$. This implies that there exists a constant $c(\epsilon, \eta, \gamma)>0$ such that

$$
\hat{\mathbf{P}}_{+, \lambda}^{r}\left(\underline{x}^{\lambda}(1-\gamma) \in O_{\epsilon}(\underline{z}), \max _{t \leq 1-\gamma} x_{n}^{\lambda}(t) \leq 2 \eta\right) \geq c(\epsilon, \eta, \gamma)
$$

for all $\underline{r}, \underline{z} \in \mathbb{A}_{n, \lambda}^{+, r}$. Since $O_{\epsilon}(\underline{z})$ is separated from the boundary of $\mathbb{A}_{n}^{+}$, we may choose $\gamma$ so small that the probability that the random walk $x^{\lambda}$ started at $y \in O_{\epsilon}(\underline{z})$ has, at time $\gamma$, the value $\underline{z}$ and leaves $\mathbb{A}_{n, \lambda}^{+}$before time $\gamma$ is quite small. This heuristic is made precise in [6, Lemma 29]. In our notation, we can state that result as follows: There exist $a>0$ and $c_{1}<\infty$ such that

$$
\begin{aligned}
\hat{\mathbf{P}}_{+, \lambda}^{\underline{y}}\left(\underline{x}^{\lambda}(\gamma)=\underline{z}, \max _{t \leq \gamma} x_{n}^{\lambda}(t)\right. & \leq 2 \eta) \\
& \geq \hat{\mathbf{P}} \frac{y}{\lambda}\left(\underline{x}^{\lambda}(\gamma)=\underline{z}, \max _{t \leq \gamma} x_{n}^{\lambda}(t) \leq 2 \eta\right)-c_{1} \gamma^{-n / 2} e^{-a \epsilon^{2} / \gamma} h_{\lambda}^{n} .
\end{aligned}
$$

By a similar argument, one can show that

$$
\hat{\mathbf{P}} \frac{y}{\lambda}\left(\underline{x}^{\lambda}(\gamma)=\underline{z}, \max _{t \leq \gamma} x_{n}^{\lambda}(t) \leq 2 \eta\right) \geq \hat{\mathbf{P}}_{\lambda}^{\underline{y}}\left(\underline{x}^{\lambda}(\gamma)=\underline{z}\right)-c_{2} \gamma^{-n / 2} e^{-a \eta^{2} / \gamma} h_{\lambda}^{n} .
$$

Finally, by the standard local limit theorem,

$$
\hat{\mathbf{P}}_{\lambda}^{\underline{y}}\left(\underline{x}^{\lambda}(\gamma)=\underline{z}\right) \geq c_{3} \gamma^{-n / 2} h_{\lambda}^{n}
$$


As a result, we have the bound

$$
\hat{\mathbf{P}}_{+, \lambda}^{\underline{y}}\left(\underline{x}^{\lambda}(\gamma)=\underline{z}, \max _{t \leq \gamma} x_{n}^{\lambda}(t) \leq 2 \eta\right) \geq c_{4} h_{\lambda}^{n},
$$

uniformly in $\underline{y}, \underline{z} \in \mathbb{A}_{n, \lambda}^{+, r}$. Combining this bound with (A.1), we infer that

$$
\hat{\mathbf{P}}_{1,+, \lambda}^{r}\left(\underline{x}^{\lambda}(1)=\underline{z}, \max _{t \leq 1} x_{n}^{\lambda}(t) \leq 2 \eta\right) \geq c_{5} h_{\lambda}^{n},
$$

uniformly in $\underline{r}, \underline{z} \in \mathbb{A}_{n, \lambda}^{+, r}$.

\section{APPENDIX B. INVARIANCE PRINCIPLES FOR RANDOM WALKS IN WEYL CHAMBERS}

Conditional limit theorems and conditional invariance principles for random walks in different cones have been studied in [6] and [8]. All the results in these papers are proved in the case when the non-rescaled walk starts at a fixed point. In this paragraph, we give certain improvements of these results to the case when the starting point of the non-rescaled walk may grow (but we shall consider walks in Weyl chambers only).

More precisely, we shall the following subsets of the euclidian space:

- chamber of type $A$ : $\left\{x: x_{1}<x_{2}<\ldots<x_{n}\right\}$;

- chamber of type $C:\left\{x: 0<x_{1}<x_{2}<\ldots<x_{n}\right\}$;

- chamber of type $D:\left\{x:\left|x_{1}\right|<x_{2}<\ldots<x_{n}\right\}$.

Let $u_{W}$ denote the unique (up to a constant multiplier) positive harmonic function on $W$ :

- if $W$ is the chamber of type $A$, then $u_{W}(x)=\prod_{i<j}\left(x_{j}-x_{i}\right)$;

- if $W$ is the chamber of type $C$, then $u_{W}(x)=\prod_{k} x_{k} \prod_{i<j}\left(x_{j}^{2}-x_{i}^{2}\right)$;

- if $W$ is the chamber of type $D$, then $u_{W}(x)=\prod_{i<j}\left(x_{j}^{2}-x_{i}^{2}\right)$.

Proposition B.1. Let $W$ be a Weyl chamber of type A, $C$ or D. Let $\tau$ be the first exit time from $W$, that is,

$$
\tau=\inf \left\{t>0: \underline{x}^{\lambda}(t) \notin W\right\} .
$$

Then, as $\underline{r}=\underline{r}_{\lambda} \rightarrow 0$,

$$
\hat{\mathbf{P}}_{\lambda}^{\underline{r}}\left(\underline{x}^{\lambda}(1) \in \cdot \mid \tau>1\right) \rightarrow \mu \quad \text { weakly, }
$$

where $\mu$ is the probability measure on $W$ with density proportional to $u_{W}(x) e^{-|x|^{2} / 2}$.

Furthermore, under $\hat{\mathbf{P}}_{\lambda}^{r}, \underline{x}^{\lambda}$ converges weakly on $C[0,1]$ to the Brownian meander in $W$ started at zero.

By "Brownian meander in $W$ ", we mean a Brownian motion conditioned on staying in $W$ up to time one. If the starting point lies inside $W$, then one has a condition of positive probability. However, if the starting point lies on the boundary of $W$, then the probability of the condition is zero and it is not at all clear how one can 
construct such a process. Garbit [11] has constructed Brownian meanders started at zero for a quite large class of cones. This class includes Weyl chambers.

Proof. The main difference with [6, Theorem 3] is that we find the limit for conditional distributions without determining the asymptotic behavior of $\hat{\mathbf{P}}_{\lambda}^{r}(\tau>1)$. (Recall once again that [6, Theorem 3] is proven under the assumption $\underline{r}=h_{\lambda}$ a for some fixed $a \in W$.)

Fix some $\epsilon \in(0,1 / 2)$ and define the stopping time

$$
\nu_{\lambda, \epsilon}=\inf \left\{t>0: \underline{x}^{\lambda}(t) \in W_{\lambda, \epsilon}\right\},
$$

where

$$
W_{\lambda, \epsilon}=\left\{x \in W: \operatorname{dist}(x, \partial W) \geq H_{\lambda}^{-2 \epsilon}\right\} .
$$

According to [6, Lemma 14],

$$
\hat{\mathbf{P}}_{\lambda}^{\frac{r}{\lambda}}\left(\tau>H_{\lambda}^{-2 \epsilon}, \nu_{\lambda, \epsilon}>H_{\lambda}^{-2 \epsilon}\right) \leq e^{-c_{1} H_{\lambda}^{2 \epsilon}},
$$

uniformly in $\underline{r}$. Since we consider lattice random walks, there exists $\underline{r}_{0}$ such that

$$
\hat{\mathbf{P}}_{\lambda}^{\frac{r}{\lambda}}(\tau>1) \geq \hat{\mathbf{P}}_{\lambda}^{\underline{r}_{0}}(\tau>1) .
$$

(If $W$ is of type $A$ or $C$, then we may take $r_{0}=h_{\lambda}(1,2, \ldots, n)$, while if $W$ is of type $D$, then we may take $r_{0}=h_{\lambda}(0,1, \ldots, n-1)$.) According to $[6$, Theorem 1],

$$
\hat{\mathbf{P}}_{\lambda}^{\underline{r}_{0}}(\tau>1) \sim C_{1} h_{\lambda}^{p}
$$

where $p$ is a positive constant depending on the type of $W$ only. Consequently,

$$
\hat{\mathbf{P}}_{\lambda}^{\frac{r}{\lambda}}(\tau>1) \geq C_{2} h_{\lambda}^{p}
$$

uniformly in $\underline{r}$. Combining (B.1) and (B.2), we infer that

$$
\frac{\hat{\mathbf{P}}_{\lambda}^{r}\left(\tau>H_{\lambda}^{-2 \epsilon}, \nu_{\lambda, \epsilon}>H_{\lambda}^{-2 \epsilon}\right)}{\hat{\mathbf{P}}_{\lambda}^{r}(\tau>1)} \rightarrow 0, \quad \lambda \downarrow 0,
$$

uniformly in $\underline{r}$. Furthermore, it follows from the exponential Doob inequality that

$$
\hat{\mathbf{P}}_{\lambda}^{r}\left(\max _{t \leq H_{\lambda}^{-2 \epsilon}}\left|\underline{x}^{\lambda}(t)-\underline{x}^{\lambda}(0)\right|>\theta_{\lambda}\right) \leq e^{-c_{2} \theta_{\lambda}^{2} H_{\lambda}^{\epsilon}}
$$

where $\theta_{\lambda} \rightarrow 0$ sufficiently slowly. This implies that, whenever $|\underline{r}| \leq \theta_{\lambda}$,

$$
\frac{\hat{\mathbf{P}}_{\lambda}^{\frac{r}{\lambda}}\left(\max _{t \leq H_{\lambda}^{-2 \epsilon}}\left|\underline{x}^{\lambda}(t)\right|>2 \theta_{\lambda}\right)}{\hat{\mathbf{P}}_{\lambda}^{r}(\tau>1)} \rightarrow 0 .
$$

It follows now from (B.3) and (B.4) that, uniformly in $\underline{r}$,

$$
\hat{\mathbf{P}}_{\lambda}^{\frac{r}{\lambda}}(\tau>1)=(1+o(1)) \hat{\mathbf{P}}_{\lambda}^{\frac{r}{\lambda}}\left(\tau>1, \nu_{\lambda, \epsilon} \leq H_{\lambda}^{-2 \epsilon}, \max _{t \leq \nu_{\lambda, \epsilon}}|\underline{x}(t)| \leq 2 \theta_{\lambda}\right)
$$


and

$$
\begin{aligned}
\hat{\mathbf{P}}_{\lambda}^{r}\left(\underline{x}^{\lambda}(1)\right. & \in A, \tau>1) \\
& =(1+o(1)) \hat{\mathbf{P}}_{\lambda}^{\frac{r}{\lambda}}\left(\underline{x}^{\lambda}(1) \in A, \tau>1, \nu_{\lambda, \epsilon} \leq H_{\lambda}^{-2 \epsilon}, \max _{t \leq \nu_{\lambda, \epsilon}}|\underline{x}(t)| \leq 2 \theta_{\lambda}\right)
\end{aligned}
$$

for any compact $A \subset W$.

Using the Markov property at time $\nu_{\lambda, \epsilon}$ and applying [6, Lemma 20], we obtain from (B.5) and (B.6)

$$
\hat{\mathbf{P}}_{\lambda}^{\frac{r}{\lambda}}(\tau>1)=\left(c_{3}+o(1)\right) h_{\lambda}^{p} \hat{\mathbf{E}}_{\lambda}^{r}\left[u_{W}\left(\underline{x}^{\lambda}\left(\nu_{\lambda, \epsilon}\right)\right) ; \nu_{\lambda, \epsilon} \leq H_{\lambda}^{-2 \epsilon}, \max _{t \leq \nu_{\lambda, \epsilon}}|\underline{x}(t)| \leq 2 \theta_{\lambda}\right]
$$

and

$$
\begin{aligned}
\hat{\mathbf{P}}_{\lambda}^{r}\left(\underline{x}^{\lambda}(1) \in A, \tau>1\right)=\left(c_{4}\right. & +o(1)) h_{\lambda}^{p} \int_{A} u_{W}(z) e^{-|z|^{2} / 2} \mathrm{~d} z \\
& \times \hat{\mathbf{E}}_{\lambda}^{\frac{r}{\lambda}}\left[u_{W}\left(\underline{x}^{\lambda}\left(\nu_{\lambda, \epsilon}\right)\right) ; \nu_{\lambda, \epsilon} \leq H_{\lambda}^{-2 \epsilon}, \max _{t \leq \nu_{\lambda, \epsilon}}|\underline{x}(t)| \leq 2 \theta_{\lambda}\right] .
\end{aligned}
$$

Thus, the proof of the first statement is completed.

To prove the functional convergence, it suffices to repeat the proof of $[8$, Theorem 1] using (B.3) and (B.4) instead of the corresponding estimates therein.

Corollary B.1. Let $W$ be the chamber of type C. If $\underline{r}=\underline{r}_{\lambda} \rightarrow \underline{r}^{*} \in \partial W$, then the sequence $\hat{\mathbf{P}}_{\lambda}^{r}\left(\underline{x}^{\lambda}(1) \in A \mid \tau>1\right)$ converges weakly. The densities of limiting laws on $W$ has are uniformly bounded. Moreover, $\underline{x}^{\lambda}$ converges weakly on $C[0,1]$ towards the Brownian meander in $W$ started at $\underline{r}^{*}$.

Proof. We just split the original set of random walks into a finite number of subsets in such a way that the differences of coordinates of the starting points in every block converge to zero and the differences of coordinates from different blocks stay bounded away from zero. Then, the probability that different blocks do not intersect is bounded away from zero and, consequently, the conditioning on $\{\tau>1\}$ is equivalent to conditioning every block on staying in the corresponding chamber. (If $r_{1}^{*}>0$, then every block is a random block in a chamber of type $A$, while if $r_{1}^{*}=0$, then the lowest block is a random walk in a chamber of type $C$ and all other blocks are random walks in chambers of type $A$.)

Proof of (I.2). Assume that there exists a sequence $\underline{r}(j)$ such that

$$
\hat{\mathbf{P}}_{1, \lambda}^{r(j)}\left(\max _{t \leq 1} x_{n}^{\lambda}(t) \leq 2 \eta \mid \tau>1\right) \rightarrow 0 .
$$

Since we are looking at starting points $\underline{r}$ with $r_{n} \leq \eta$, there exists a convergent subsequence $\underline{r}\left(j_{k}\right)$. Let $\underline{r}^{*}$ denote the limiting point. It follows immediately from the usual functional CLT that the case $\underline{r}^{*} \in W$ is impossible. But, if $\underline{r}^{*} \in \partial W$, then we may use Corollary B.1 to conclude that the Brownian meander in $W$ started at 
$\underline{r}^{*}$ leaves the set $\left\{\underline{x} \in W: x_{n} \leq 2 \eta\right\}$ with probability one. However, this would contradict [18, Theorem 3.2]. Thus,

$$
\inf _{\underline{r}: r_{n} \leq \eta} \hat{\mathbf{P}}_{1, \lambda}^{r}\left(\max _{t \leq 1} x_{n}^{\lambda}(t) \leq 2 \eta \mid \tau>1\right)>0
$$

which implies (I.2).

Proof of (I.3). Fix some $\epsilon>0$ and define

$$
W_{\leq \epsilon}=\left\{\underline{x} \in W:\left|x_{i+1}-x_{i}\right| \leq \epsilon \text { for some } i \geq 0\right\} .
$$

Assume that there exists a sequence $\underline{r}(j)$ such that

$$
\hat{\mathbf{P}}_{1, \lambda}^{r(j)}\left(\underline{x}^{\lambda}(1) \in W_{\leq \epsilon} ; \max _{t \leq 1} x_{n}^{\lambda}(t) \leq 2 \eta \mid \tau>1\right) \geq \epsilon^{1 / 2} .
$$

We may again assume that $\underline{r}(j)$ converges to $\underline{r}^{*}$ and this limiting point can not lie in $W$. But, if $\underline{r}^{*}$ is on the boundary of $W$, then the conditions of Corollary B.1 are satisfied and the contradiction follows now from the boundedness of the density of the limiting law and the fact that $\operatorname{vol}\left(W_{\leq \epsilon} \cap\left\{\underline{x}: x_{n} \leq 2 \eta\right\}\right) \leq C_{3} \eta^{n-1} \epsilon$.

As a consequence we have that, for all $\epsilon$ small enough,

$$
\inf _{\underline{r}: r_{n} \leq \eta} \hat{\mathbf{P}}_{1,+, \lambda}^{r}\left(\underline{x}^{\lambda}(1) \in \mathbb{A}_{n, \lambda}^{+, r}, \max _{t \leq 1} x_{n}^{\lambda}(t) \leq 2 \eta \mid \tau>1\right) \geq 1-\epsilon^{1 / 2} .
$$

Combining (B.7) and (B.8), we conclude that (I.3) holds for $t=1$ and all $\epsilon$ sufficiently small. Using Brownian scaling, we conclude that (I.3) is valid for all $t>0$.

\section{REFERENCES}

[1] P. Billingsley. Convergence of probability measures. John Wiley \& Sons, Inc., New YorkLondon-Sydney, 1968.

[2] F. Bornemann. On the scaling limits of determinantal point processes with kernels induced by Sturm-Liouville operators. Preprint, arXiv:1104.0153, 2011.

[3] P. Caputo, E. Lubetzky, F. Martinelli, A. Sly, and F. L. Toninelli. Scaling limit and cube-root fluctuations in sos surfaces above a wall. Preprint, arXiv:1302.6941, 2013.

[4] P. Caputo, F. Martinelli, and F. L. Toninelli. On the probability of staying above a wall for the (2+1)-dimensional sos model at low temperature. Preprint, arXiv:1406.1206, 2014.

[5] E. A. Coddington and N. Levinson. Theory of ordinary differential equations. McGraw-Hill Book Company, Inc., New York-Toronto-London, 1955.

[6] D. Denisov and V. Wachtel. Random walks in cones. Ann. Probab., 43(3):992-1044, 2015.

[7] M. Duits. On global fluctuations for non-colliding processes. Preprint, arXiv:1510.08248.

[8] J. Duraj and V. Wachtel. Invariance principles for random walks in cones. Preprint, arXiv:1508.07966, 2015.

[9] C. G. Esseen. On the concentration function of a sum of independent random variables. $Z$. Wahrscheinlichkeitstheorie und Verw. Gebiete, 9:290-308, 1968.

[10] P. L. Ferrari and H. Spohn. Constrained Brownian motion: fluctuations away from circular and parabolic barriers. Ann. Probab., 33(4):1302-1325, 2005.

[11] R. Garbit. Brownian motion conditioned to stay in a cone. J. Math. Kyoto Univ., 49:573-592, 2009. 
[12] O. Hryniv and Y. Velenik. Some rigorous results on semiflexible polymers. I. Free and confined polymers. Stochastic Process. Appl., 119(10):3081-3100, 2009.

[13] D. Ioffe and S. Shlosman. Ising model fog drip: the first two droplets. In In and out of equilibrium. 2, volume 60 of Progr. Probab., pages 365-381. Birkhäuser, Basel, 2008.

[14] D. Ioffe, S. Shlosman, and F. L. Toninelli. Interaction versus entropic repulsion for low temperature Ising polymers. J. Stat. Phys., 158(5):1007-1050, 2015.

[15] D. Ioffe, S. Shlosman, and Y. Velenik. An invariance principle to Ferrari-Spohn diffusions. Comm. Math. Phys., 336(2):905-932, 2015.

[16] D. Ioffe and Y. Velenik. Ballistic phase of self-interacting random walks. In Analysis and stochastics of growth processes and interface models, pages 55-79. Oxford Univ. Press, Oxford, 2008.

[17] S. Karlin and J. McGregor. Coincidence probabilities. Pacific J. Math., 9:1141-1164, 1959.

[18] W. König and P. Schmid. Brownian motion in a truncated Weyl chamber. Markov Process. Related Fields, 17(4):499-522, 2011.

[19] A. Soshnikov. Determinantal random point fields. Uspekhi Mat. Nauk, 55(5(335)):107-160, 2000.

[20] K. Uchiyama. One dimensional lattice random walks with absorption at a point/on a half line. J. Math. Soc. Japan, 63(2):675-713, 2011.

[21] Y. Velenik. Entropic repulsion of an interface in an external field. Probab. Theory Related Fields, 129(1):83-112, 2004.

Faculty of IE\&M, Technion, Haifa 32000, IsRael

E-mail address: ieioffe@ie.technion.ac.il

Section de Mathématiques, Université de Genève, CH-1211 Genève, Switzerland E-mail address: yvan.velenik@unige.ch

Institut für Mathematik, Universität Augsburg, D-86135 Augsburg, Germany

E-mail address: vitali.wachtel@math.uni-augsburg.de 